\title{
Multimodal Metabolic Imaging Reveals Pigment Reduction and Lipid Accumulation in Metastatic Melanoma
}

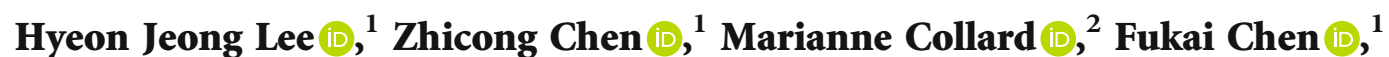 \\ Jiaji G. Chen $\mathbb{D}^{1},{ }^{2}$ Muzhou Wu, ${ }^{2}$ Rhoda M. Alani $\mathbb{D}^{2},{ }^{2}$ and Ji-Xin Cheng $\mathbb{D}^{1}$ \\ ${ }^{1}$ Photonics Center, Department of Electrical and Computer Engineering, Department of Biomedical Engineering, Boston University, \\ Boston, MA 02215, USA \\ ${ }^{2}$ Department of Dermatology, Boston University School of Medicine, Boston, MA 02118, USA
}

Correspondence should be addressed to Rhoda M. Alani; alani@bu.edu and Ji-Xin Cheng; jxcheng@bu.edu

Received 15 March 2021; Accepted 30 August 2021; Published 8 October 2021

Copyright (C) 2021 Hyeon Jeong Lee et al. Exclusive Licensee Suzhou Institute of Biomedical Engineering and Technology, CAS. Distributed under a Creative Commons Attribution License (CC BY 4.0).

Objective and Impact Statement. Molecular signatures are needed for early diagnosis and improved treatment of metastatic melanoma. By high-resolution multimodal chemical imaging of human melanoma samples, we identify a metabolic reprogramming from pigmentation to lipid droplet (LD) accumulation in metastatic melanoma. Introduction. Metabolic plasticity promotes cancer survival and metastasis, which promises to serve as a prognostic marker and/or therapeutic target. However, identifying metabolic alterations has been challenged by difficulties in mapping localized metabolites with high spatial resolution. Methods. We developed a multimodal stimulated Raman scattering and pump-probe imaging platform. By time-domain measurement and phasor analysis, our platform allows simultaneous mapping of lipids and pigments at a subcellular level. Furthermore, we identify the sources of these metabolic signatures by tracking deuterium metabolites at a subcellular level. By validation with mass spectrometry, a specific fatty acid desaturase pathway was identified. Results. We identified metabolic reprogramming from a pigment-containing phenotype in low-grade melanoma to an LD-rich phenotype in metastatic melanoma. The LDs contain high levels of cholesteryl ester and unsaturated fatty acids. Elevated fatty acid uptake, but not de novo lipogenesis, contributes to the LD-rich phenotype. Monounsaturated sapienate, mediated by FADS2, is identified as an essential fatty acid that promotes cancer migration. Blocking such metabolic signatures effectively suppresses the migration capacity both in vitro and in vivo. Conclusion. By multimodal spectroscopic imaging and lipidomic analysis, the current study reveals lipid accumulation, mediated by fatty acid uptake, as a metabolic signature that can be harnessed for early diagnosis and improved treatment of metastatic melanoma.

\section{Introduction}

Melanoma is the most aggressive form of skin cancer. When melanoma is caught in its early stages and surgically removed, the prognosis is favorable; once melanoma has metastasized, it becomes difficult to treat $[1,2]$. Although increasing Breslow depth, or melanoma tumor thickness, is associated with worse survival [3], the majority of deaths attributed to melanoma are due to thin tumors $(<1 \mathrm{~mm})$ [4]. With the incidence of melanoma steadily rising at a rate of 3\% per year [5], it is more important than ever to detect melanoma at an early stage and identify the tumors that are the most at-risk for metastasizing for early intervention. Nevertheless, without molecular signatures, precision diagnosis and treatment of metastatic melanoma remain difficult.
One promising target is melanin, the major pigment in melanoma. It was found that more eumelanin than pheomelanin is present in malignant melanoma compared to dysplastic nevi $[6,7]$. A recent study used pump-probe microscopy as an elegant label-free approach to distinguish the two types of melanin in melanoma tissues [8], heralding potential prognostic significance [9]. Despite this advance, loss of microphthalmia-associated transcription factor (MITF), the gene regulating pigmentation, is reported in melanomas with an invasive phenotype $[10,11]$, indicating an unmet need to identify new molecular markers for detecting aggressive and invasive melanoma.

Melanoma exhibits a dynamic and adaptive phenotypic switching capability [12]. Among the signaling pathways mediating this process, the MITF status is considered a 
central regulator of the phenotypic switch [13], namely, from a highly proliferative, less invasive to a less proliferative, highly invasive state. Specifically, loss of MITF is an indication of dedifferentiation of melanoma [14, 15], which is supported by enhanced migration/invasion gene expression profiles [16]. Despite these advances in the genetic characterization of melanoma phenotypes, metabolic markers for advanced melanoma have just begun to be explored. A recent report suggested elevated lipid uptake in metastatic melanoma [17], indicating that cancer progression is partly sustained by high lipogenic or lipid uptake activity; however, due to difficulties in compositional analysis, the exact metabolites and precise mechanisms that regulate melanoma progression remain unknown.

Here, we report findings of metabolic reprogramming in metastatic melanoma made by a multimodal chemical imaging platform. Specifically, by coregistered stimulated Raman scattering (SRS) imaging of biomolecules [18, 19] and pump-probe imaging of pigments [20] at a subcellular level, we revealed a previously unrecognized metabolic reprogramming from pigmentation in low-grade melanoma to lipid droplet (LD) accumulation in metastatic melanoma. Raman spectroscopy and lipidomic analysis further identified a significant amount of unsaturated fatty acids and cholesteryl ester (CE) in these LDs. Blocking fatty acid uptake and depleting LDs dramatically suppresses cell migration. In particular, the unsaturated fatty acid, sapienate, synthesized through fatty acid desaturase 2 (FADS2), is found to play an essential role in promoting melanoma migration, which may be mediated through membrane fluidity regulation. Consequently, inhibition of FADS2 suppresses melanoma migration in vitro and metastasis in vivo. In parallel, inhibiting cholesterol esterification is found to significantly reduce LD accumulation and suppress cell migration by inactivation of the $\mathrm{Wnt} / \beta$-catenin pathway. Together, these results present a unique metabolic reprogramming profile that can be used for molecular diagnosis and treatment of metastatic melanoma.

\section{Results}

2.1. Melanoma Progression into Metastatic State Is Accompanied by Metabolic Reprogramming from Pigmentation to Lipid Accumulation. To investigate the metabolic reprogramming during melanoma progression, human melanoma cell lines bearing high MITF or low MITF expression were used. Consistent with previous reports [11], the melanoma cells with high MITF expression levels exhibit low AXL Receptor Tyrosine Kinase (AXL) expression (Figure S1a, b). On the basis of this expression profile, the human melanoma cell lines used in this study (Table S1) were clustered into $\mathrm{MITF}^{\text {high }} / \mathrm{AXL}^{\text {low }}$ or $\mathrm{MITF}^{\text {low }} / \mathrm{AXL}^{\text {high }}$ groups. To map the various metabolites at sub-cellular resolution in human melanoma cells, we deployed stimulated Raman scattering microscopy [18, 19]. The laser beating frequency was initially tuned to be resonant with C$\mathrm{H}$ stretching vibration at $2899 \mathrm{~cm}^{-1}$. We detected a stimulated Raman loss signal arising from C-H-rich biomolecules, showing a clear cell morphology with a number of droplets in melanocytes and $\mathrm{MITF}^{\text {high }} / \mathrm{AXL}^{\text {low }}$ and MITF $^{\text {low }} /$ AXL $^{\text {high }}$ melanoma cells (Figure $1(\mathrm{a})$ ). These droplet structures in an SRS image are often considered to be lipid droplets (LDs) [18]. To verify the chemical specificity, we tuned the laser beating frequency to $2265 \mathrm{~cm}^{-}$ ${ }^{1}$, off-resonant with $\mathrm{C}-\mathrm{H}$ stretching vibration. No signal was observed from the off-resonance image of $\mathrm{MITF}^{\text {low }} / \mathrm{AXL}^{\text {high }}$ melanoma cells, which suggests that these droplets are rich in $\mathrm{C}-\mathrm{H}$ bonds and are likely LDs (Figure 1(a)). This assignment was confirmed by immunofluorescence staining of an LD-associated protein, adipophilin (Figure S1c).

Intriguingly, droplets with strong signals were still observed off the Raman resonance in melanocytes and $\mathrm{MITF}^{\text {high }} / \mathrm{AXL}^{\text {low }}$ melanoma, indicating that signals from these droplets are not specific to $\mathrm{C}-\mathrm{H}$ stretching vibration (Figure 1(a)). We attribute the strong signal at off-Raman resonance to either transient absorption or photothermal effects, which are often detected with a pump-probe imaging scheme [21]. To verify this hypothesis, we performed a timeresolved measurement. The signals from the droplets in $\mathrm{MITF}^{\text {high }} / \mathrm{AXL}^{\text {low }}$ melanoma show a fast decay within 1.0 picosecond and then remained at the same level (Figure S1d). The fast decay and the remaining signal are attributed to transient absorption and photothermal processes, respectively [22]. In comparison, the pump-probe decay curve of eumelanin powder extracted from Sepia officinalis showed a similar profile (Figure S1e). Unlike the pigments in melanoma, the pigments from melanocytes exhibit a negative decay curve (Figure S1d), indicating a different type of pigment in melanocytes. It was reported that when imaged under a pump-probe microscope, pheomelanin exhibits a negative signal whereas eumelanin exhibits a positive signal [8]. Thus, it is likely that the pigments in melanoma are mostly eumelanin and the pigments in melanocytes are mostly pheomelanin. Consistent with this observation, it was reported that eumelanin is presented in higher content in melanoma compared to nonmalignant nevi $[6,8]$. Collectively, these results support that both melanocytes and MITF ${ }^{\text {high }} / \mathrm{AXL}^{\text {low }}$ melanoma cells have high pigmentation activity, concordant with high MITF expression.

To establish a method for quantifying pigmentation and LD accumulation in melanoma, we designed a time-domain multimodal SRS/pump-probe imaging and phasor analysis approach (Figure S2). In SRS imaging, $802 \mathrm{~nm}$ serves as a pump beam and $1045 \mathrm{~nm}$ serves as a Stokes beam, while in pump-probe imaging, $1045 \mathrm{~nm}$ serves as a pump beam and $802 \mathrm{~nm}$ serves as a probe beam (Figure S2a). SRS and pump-probe signals show different profiles in the timedomain: SRS displays a sharp Gaussian-shape peak, while pump-probe displays a slow decay curve (Figure S2b, c). These two signals can be well-separated by phasor analysis of the time-resolved measurements [23] (Figure S2d). Furthermore, we found that the subtle SRS spectral difference between LDs versus the rest of the cell can be further separated through phasor analysis (Figure S2d). Therefore, using the multimodal imaging and phasor analysis approach, we simultaneously imaged and quantified LD accumulation and pigmentation in human melanoma cell lines (Figure 1(b) and Figure S2e, f). There 


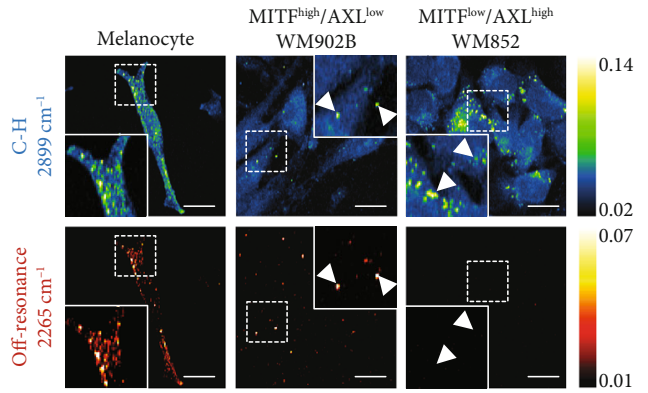

(a)

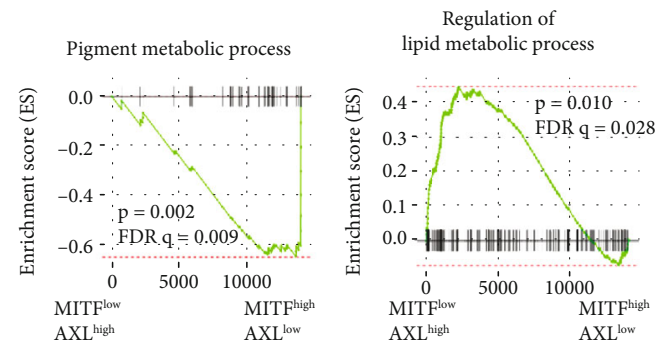

(c)
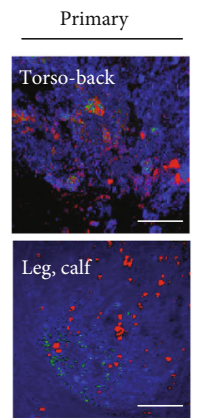

- $\mathrm{LD}$

- Pigment

- Other biomolecules

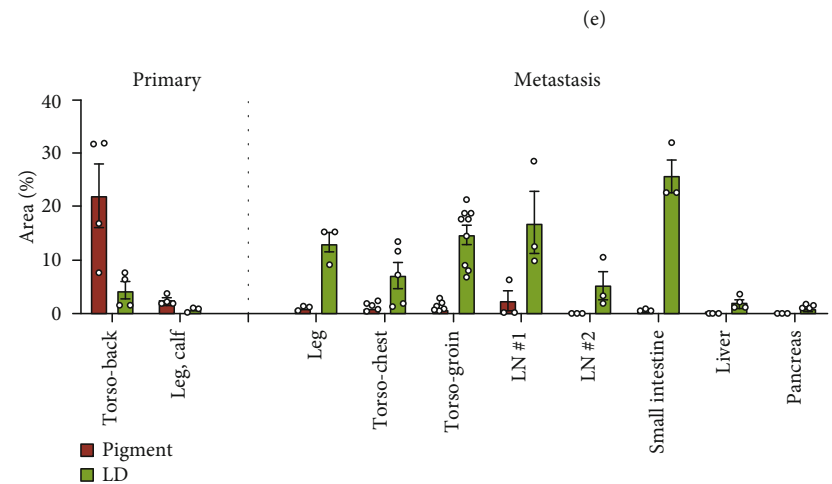

(f)

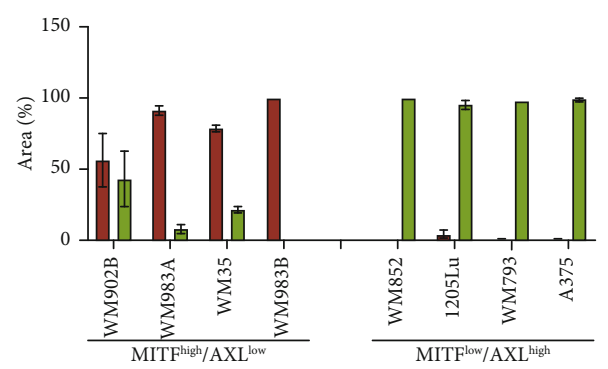

口 Pigment/total droplets 口 LD/total droplets

(b)

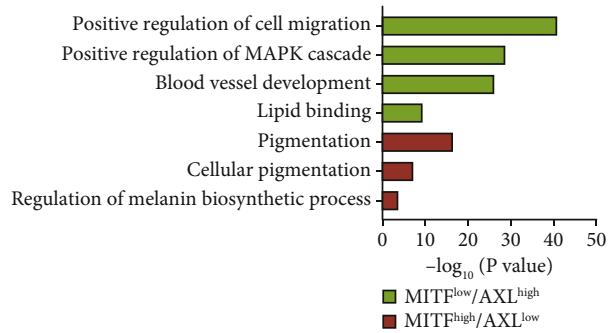

(d)

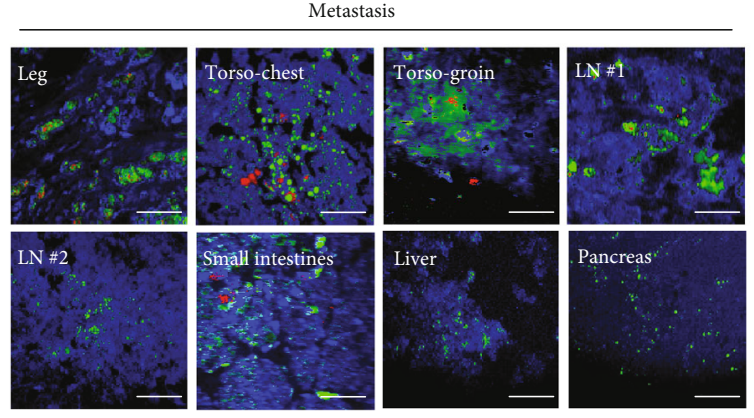


is a high percentage of pigment droplets in $\mathrm{MITF}^{\text {high }} / \mathrm{AXL}^{\text {low }}$ melanoma. Nevertheless, almost all droplets identified in $\mathrm{MITF}^{\text {low }} / \mathrm{AXL}{ }^{\text {high }}$ melanoma are LDs (Figure 1(b)). Together, these data indicate a reduction of pigmentation and increase of lipid accumulation during the progression of melanoma from low grade to high grade.

Consistent with the metabolic profile observed in the cell lines, the Gene Set Enrichment Analysis (GSEA) of human melanoma samples from The Cancer Genome Atlas (TCGA) showed that the pigment metabolic process gene set was enriched in the $\mathrm{MITF}^{\text {high }} / \mathrm{AXL}^{\text {low }}$ group, while regulation of the lipid metabolic process gene set was enriched in the $\mathrm{MITF}^{\text {low }} / \mathrm{AXL}^{\text {high }}$ group (Figure 1(c) and Table 1(c) and Table S2). Differentially expressed genes (DEGs) were generated based on the median expression level of MITF and AXL (Figure S3a and Table S3). Functional enrichment network analysis for MITF $^{\text {low }} /$ AXL $^{\text {high }}$ melanoma identified several gene sets related to aspects of cancer metastasis, such as cell migration and blood vessel development and lipid binding processes that were enriched from the upregulated genes, while gene sets related to pigmentation were enriched from the downregulated genes (Figure 1(d), Figure S3b, and Table S3). These results indicate a distinct metabolic profile of $\mathrm{MITF}^{\text {high }} / \mathrm{AXI}^{\text {low }}$ versus $\mathrm{MITF}^{\text {low }} / \mathrm{AXL}^{\text {high }}$ melanoma.

To validate the clinical relevance of such metabolic reprogramming in metastatic melanoma, we performed multimodal imaging of two primary and eight metastatic melanoma tissues from deidentified human melanoma patients (Figure 1(e)). The tumor lesions were confirmed by pathological assessment of the neighboring slices stained with hematoxylin and eosin (Figure S4). By time-resolved multimodal imaging and phasor analysis, signals from pigment, LDs, and other biomolecules were separated and quantified (Figures 1(e) and 1(f)). Primary melanoma tissues contain higher pigment content than LD content. In contrast, metastatic melanomas, including metastatic lesions on the extreme leg, torso-chest, and torsogroin, and metastatic tumors in other organs such as the lymph nodes, small intestines, liver, and pancreas, contain higher LD content than pigment content (Figure 1(f)). These results collectively suggest a metabolic reprogramming from pigmentation to lipid accumulation during the progression of human melanoma from the primary tumor to the metastatic tumor (Figure 1(g)).

2.2. The LDs in MITF $F^{\text {low }} / A X L^{\text {high }}$ Melanoma Contain CE and Unsaturated Fatty Acids. To unveil the composition of LDs accumulated in MITF $^{\text {low }} / \mathrm{AXL}^{\text {high }}$ melanoma, we performed confocal Raman spectroscopic measurement of single LDs (Figure 2(a)). The Raman spectra of LDs in all $\mathrm{MITF}^{\text {low }} / \mathrm{AXL}-$ high melanoma cells showed similar spectral profiles, including bands for lipids in the fingerprint region $\left(1200-1800 \mathrm{~cm}^{-1}\right)$ and prominent $\mathrm{CH}_{2}$ stretching band $\left(2850 \mathrm{~cm}^{-1}\right)$. More detailed spectral analysis revealed that these LDs show peaks from the $\mathrm{C}=\mathrm{C}$ stretching vibration at $1654 \mathrm{~cm}^{-1}$ and the vibration of $=\mathrm{C}-\mathrm{H}$ bonds at $3002 \mathrm{~cm}^{-1}$, both suggesting the presence of unsaturated fatty acids (Figure 2(a)). To quantify the unsaturation degree, we generated a calibration curve using fatty acids containing various numbers of $\mathrm{C}=\mathrm{C}$ bonds (Figure S5a). Raman spectra of palmitate (no $\mathrm{C}=\mathrm{C}$ bond), oleate (one $\mathrm{C}=\mathrm{C}$ bond), and linoleate (two $\mathrm{C}=\mathrm{C}$ bonds) were obtained and analyzed. The peak intensity of $\mathrm{C}=\mathrm{C}$ stretching vibration at $1654 \mathrm{~cm}^{-1}$ is found to be linearly correlated with the number of $\mathrm{C}=\mathrm{C}$ bonds present in the fatty acids after normalization by the $\mathrm{CH}_{2}$ bending band at $1445 \mathrm{~cm}^{-1}$ (Figure S5a, b). On the basis of this calibration, we estimated the unsaturation degree to be 1.2 in WM852, 1.3 in $1205 \mathrm{Lu}$, 1.4 in WM793, and 1.8 in A375 LD-rich cells (Figure 2(b)). To validate the presence of unsaturated fatty acids in LDs, we treated LD-rich melanoma cells with desaturase inhibitors, CAY10566 and SC26196, for inhibiting StearoylCoA desaturase-1 (SCD) and FADS2, respectively. Cells treated with either the SCD or FADS2 inhibitor showed reduced peak intensities at both $1654 \mathrm{~cm}^{-1}$ and $3002 \mathrm{~cm}^{-1}$ (Figure 2(c)), resulting in the unsaturation degree of below or close to 1.0 (Figure 2(d)). Furthermore, the multiple reaction monitoring (MRM) profiling of neutral lipids from $\mathrm{MITF}^{\text {low }} / \mathrm{AXL}{ }^{\text {high }}$ melanoma cells shows a high level of monounsaturated fatty acids in triglycerides (Figure S5c). Together, these results indicate that the LDs accumulated in MITF $^{\text {low }} / \mathrm{AXL}^{\text {high }}$ melanoma contain a significant amount of unsaturated fatty acids.

Another important component that we identified from the Raman spectral profile is cholesteryl ester (CE). This component was indicated by the cholesterol band at $702 \mathrm{~cm}^{-1}$ and the $\mathrm{C}=\mathrm{O}$ ester stretching band at $1740 \mathrm{~cm}^{-1}$ (Figure 2(a)). To quantify the CE percentage, we generated calibration curves for $\mathrm{CE}$ percentage using $\mathrm{CE}$ and triacylglycerol emulsions (Figure S5d, e). The Raman spectra of lipid emulsions with various ratios of $\mathrm{CE}$ and triacylglycerol showed that the peak intensity of the cholesterol band at $702 \mathrm{~cm}^{-1}$ was linearly correlated to the molar percentage of $\mathrm{CE}$ in the lipid emulsions after normalization by the $\mathrm{CH}_{2}$ bending band at $1445 \mathrm{~cm}^{-1}$ (Figure S5d, e). On the basis of the calibration, it was estimated that the CE contents of the LDs are $37.3 \%$, $47.3 \%, 40.6 \%$, and $25.0 \%$ in WM852, 1205Lu, WM793, and A375, respectively (Figure 2(b)). Lipidomic analysis by MRM profiling shows similar CE percentage in neutral lipids (Figure S5f). Inside cells, excess cholesterol is known to be esterified by the sterol O-acyltransferase (SOAT) enzyme and stored in LDs [24]. To validate that CE is indeed stored in LDs, we treated cells with a potent SOAT inhibitor, avasimibe. After avasimibe treatment, the peak intensity at $702 \mathrm{~cm}^{-1}$ reduced significantly (Figure $2(\mathrm{e})$ ), indicating less than 20\% CE in the LDs (Figure 2(f)). Furthermore, the LC/MS measurement of extracted lipids from MITF $^{\text {low }} / \mathrm{AXL}^{\text {high }}$ melanoma cells confirmed the CErich lipid profile and significant reduction of multiple $\mathrm{CE}$ species after avasimibe treatment (Figure 2(g) and Figure S5g). The mass spectrometry data identified cholesteryl oleate (CE 18:1) to be the dominant species. Together, these results indicate that LDs accumulated in $\mathrm{MITF}^{\text {low }} / \mathrm{AXL}^{\text {high }}$ melanoma contain an elevated level of unsaturated fatty acids in the form of triglyceride and CE.

2.3. Fatty Acid Uptake Is the Major Pathway for LD Accumulation in MITF ${ }^{\text {low }} / A X L^{\text {high }}$ Melanoma. There are two major routes for lipid accumulation in mammalian cells: 


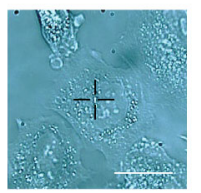

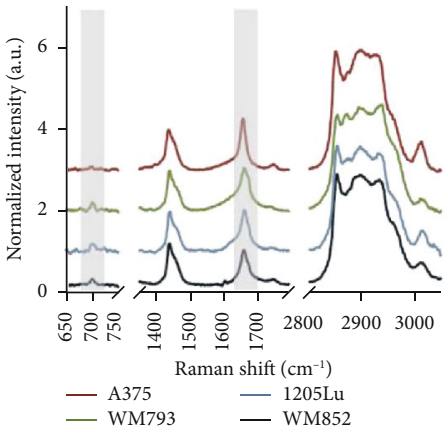

(a)

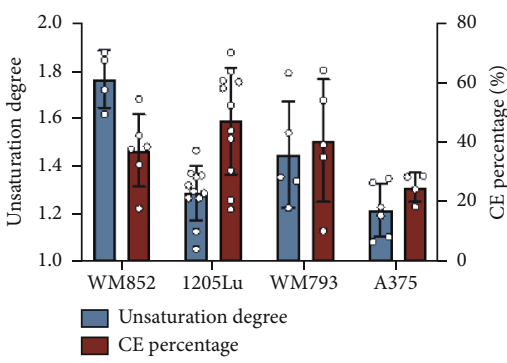

(b)

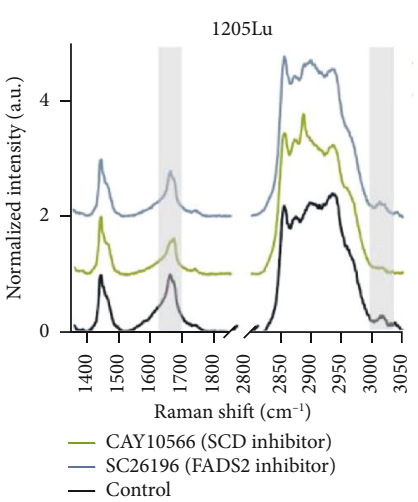

(c)

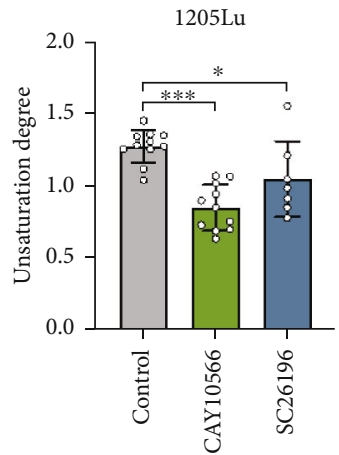

(d)

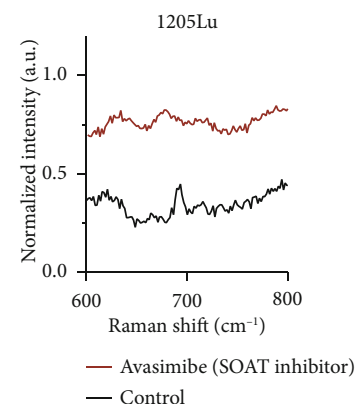

(e)

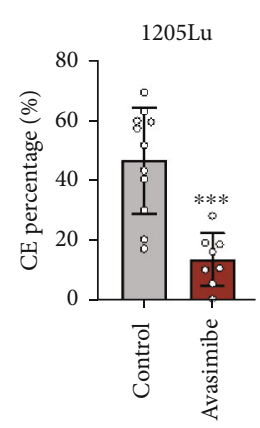

(f)

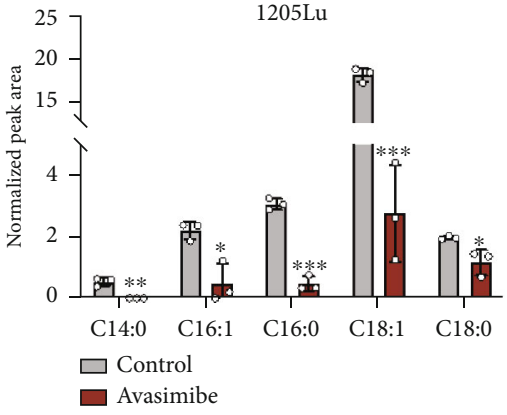

(g)

FIGURE 2: LDs in aggressive melanoma contain unsaturated fatty acids and CE. (a) Representative Raman spectra of single LDs in $\mathrm{MITF}^{\text {low }} / \mathrm{AXL}^{\text {high }}$ melanoma. The spectral intensity was normalized by the peak at $1445 \mathrm{~cm}^{-1}$. Grey areas highlight the Raman peaks used to quantify unsaturation degree and CE percentage. Insert shows the brightfield image of single LD identified in a cell. Scale bar: $10 \mu \mathrm{m}$. (b) Quantification of unsaturation degree and CE percentage based on the calibration curves. $n=4-11$. (c) Representative Raman spectra of LDs in 1205Lu treated with DMSO as control, CAY10566 (50 $\mu \mathrm{M}, 2$ days), and SC26196 (50 $\mu \mathrm{M}, 2$ days). Grey areas highlight the Raman peaks indicative of unsaturation lipids. (d) Quantification of unsaturation degree calculated from the calibration curve. $n=7$ - 11. (e) Representative Raman spectra of LDs in $1205 \mathrm{Lu}$ treated with DMSO as control and avasimibe (10 $\mu \mathrm{M}, 2$ days). (f) Quantification of CE percentage in LDs calculated from the calibration curve. $n=8-11$. (g) Quantification of LC/MS measurement of lipids extracted from control and avasimibe-treated $1205 \mathrm{Lu}$ cells $\left(10 \mu \mathrm{M}, 2\right.$ days). $n=3$ biological replicates. Data represent mean \pm SD. ${ }^{*}$ $p<0.05,{ }^{* *} p<0.01$, and ${ }^{* * *} p<0.001$.

one is de novo lipogenesis and the other is fatty acid uptake through transporter proteins (Figure 3(a)). In de novo lipogenesis, glucose typically serves as the precursor, from which the acetyl-CoA is generated and used for lipid synthesis. Fatty acid uptake is facilitated by various fatty acid transporters, such as FABP4, CD36, and FATPs. In both cases, excess fatty acids are stored in LDs. To understand how $\mathrm{MITF}^{\text {low }} / \mathrm{AXL}^{\text {high }}$ melanoma cells obtain the lipids stored in LDs, we performed SRS imaging of deuterated metabolites in melanoma cells to evaluate their glucose-derived lipogenesis and fatty acid uptake activities. As SRS imaging of glu$\operatorname{cose}-\mathrm{D}_{7}$ has been used to track the de novo lipogenesis 

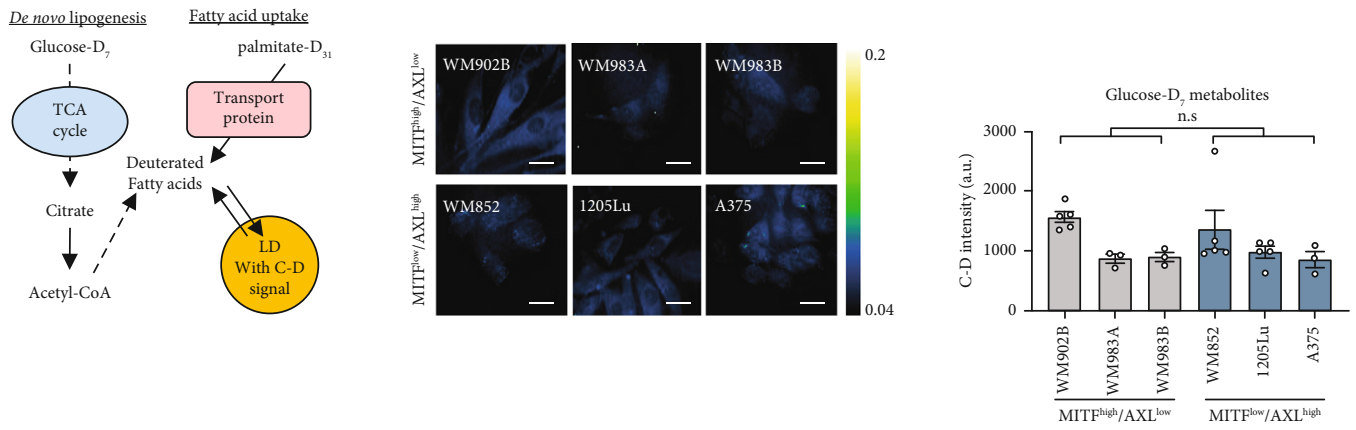

(a)

(b)

(c)

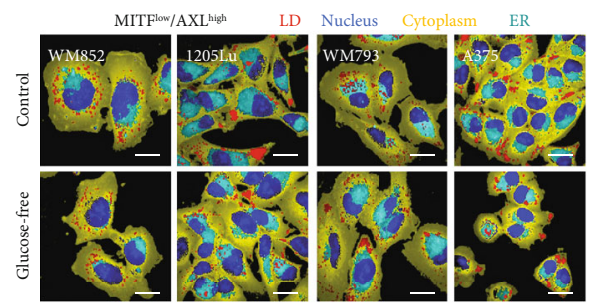

(d)
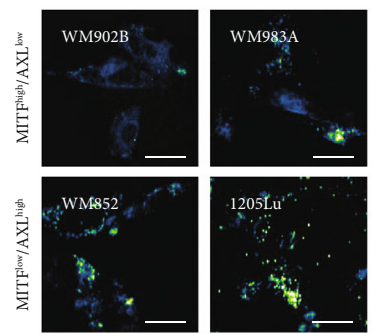

$(\mathrm{f})$
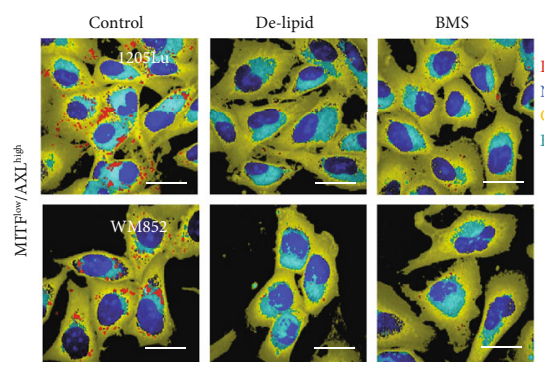

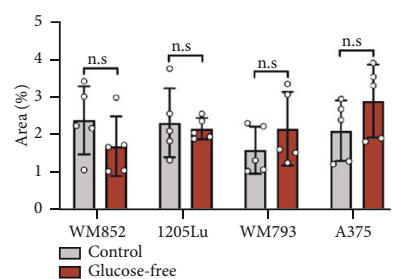

(e)
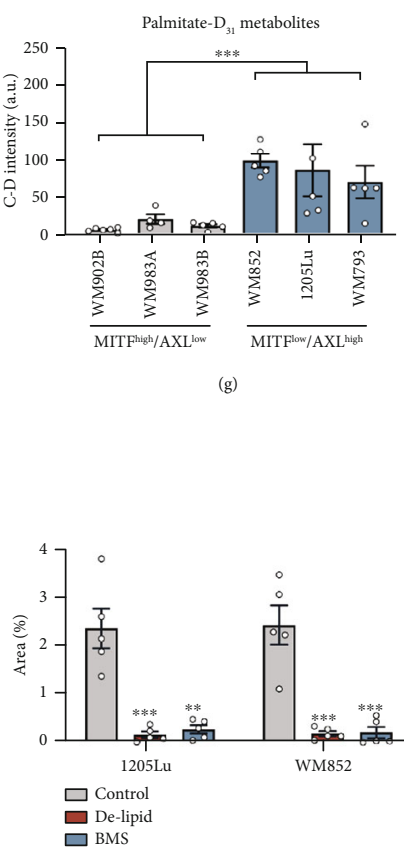

FIGURE 3: Fatty acid uptake is the major route for LD accumulation in MITF ${ }^{\text {low }} / \mathrm{AXL}^{\text {high }}$ melanoma. (a) A schematic of lipid sources contributing to LD synthesis and deuterated metabolite tracking method. (b) Representative SRS images in the C-D region $\left(2127 \mathrm{~cm}^{-1}\right.$ ) of $\mathrm{MITF}^{\text {high }} / \mathrm{AXL}^{\text {low }}$ and MITF ${ }^{\text {low }} / \mathrm{AXL}^{\text {high }}$ cells cultured with glucose- $\mathrm{D}_{7}$ containing media for 2 days. (c) Quantification of SRS intensity at $2127 \mathrm{~cm}^{-1}$ in cells $\left(n=3-5\right.$ fields of view). (d) Representative phasor output from SRS images of MITF ${ }^{\text {low }} / \mathrm{AXL}^{\text {high }}$ cells, cultured in normal media as control and in glucose-free media (2 days). (e) Percentage area of LDs in MITF ${ }^{\text {low }} / \mathrm{AXL}^{\text {high }}$ cells $(n=5$ fields of view). (f ) Representative SRS images in the C-D region $\left(2127 \mathrm{~cm}^{-1}\right)$ of WM902B, WM983A, WM983B, WM852, 1205Lu, and WM793, cultured with palmitate- $\mathrm{D}_{31}$ containing media $\left(100 \mu \mathrm{M}, 6\right.$ hours). (g) Quantification of SRS intensity at $2127 \mathrm{~cm}^{-1}$ for C-D positive LDs $(n=4-6$ fields of view). (h) Representative phasor output from SRS images of 1205Lu and WM793, cultured in normal media as control, in delipidized serum media (2 days), and treated with BMS (50 $\mu \mathrm{M}, 1$ day). (i) Percentage area of LDs in 1205Lu and WM793 ( $n=5$ fields of view). Scale bars, $10 \mu \mathrm{m}$. Data represent mean \pm SD. ${ }^{*} p<0.05,{ }^{* *} p<0.01$, and ${ }^{* * *} p<0.001$. n.s.: not significant. 
activity of cancer cells [25], we cultured $\mathrm{MITF}^{\text {high }} / \mathrm{AXL}^{\text {low }}$ and $\mathrm{MITF}^{\mathrm{low}} / \mathrm{AXL}^{\text {high }}$ melanoma cells with a glucose- $\mathrm{D}_{7^{-}}$ containing medium. SRS imaging in the $\mathrm{C}-\mathrm{D}$ vibration at $2127 \mathrm{~cm}^{-1}$ was performed to visualize glucose- $\mathrm{D}_{7}$-derived metabolites (Figure 3(b)). Interestingly, a weak signal was observed from the cytoplasm in both $\mathrm{MITF}^{\text {high }} / \mathrm{AXL}^{\text {low }}$ and MITF $^{\text {low }} / \mathrm{AXL}^{\text {high }}$ melanoma cells (Figure 3(b)). No obvious C-D-derived droplet structures were observed in both groups. The quantification of the intracellular C-D signal showed no difference between the two groups (Figure 3(c)) up to 72 hours (Figure S6a, b), indicating no significant difference in glucose-derived metabolites. To further evaluate the contribution of de novo lipogenesis to $\mathrm{LD}$ accumulation in $\mathrm{MITF}^{\text {low }} / \mathrm{AXL}^{\text {high }}$ melanoma cells, we cultured the cells in a glucose-free medium. We then performed hyperspectral SRS imaging in $\mathrm{C}-\mathrm{H}$ vibrations and quantified the LD area fraction by phasor analysis. The LD levels remained the same even without supplement of glucose (Figures 3(d) and 3(e)), indicating minimum contribution from de novo lipogenesis.

To evaluate the fatty acid uptake activity, palmitate- $\mathrm{D}_{31}$ was supplemented into the culture medium. SRS imaging at $2127 \mathrm{~cm}^{-1}$ clearly showed LDs incorporated with deuterated fatty acids, especially in MITF ${ }^{\text {low }} / \mathrm{AXL}^{\text {high }}$ melanoma cells (Figure 3(f)). To quantify LDs with the C-D signal, we performed particle analysis and measured the C-D intensity per cell area in each group. MITF ${ }^{\text {low }} / \mathrm{AXL}^{\text {high }}$ melanoma cells showed a significantly higher C-D signal than MITFhigh/AXL ${ }^{\text {low }}$ melanoma cells (Figure $3(\mathrm{~g}$ ) and Figure S6c, d), indicating high palmitate uptake activity in these cells. Similarly, when oleate- $\mathrm{D}_{34}$ was supplemented into the culture medium, MITF $^{\text {low }} / \mathrm{AXL}^{\text {high }}$ cells showed higher oleate uptake activity than $\mathrm{MITF}^{\text {high }} / \mathrm{AXL}^{\text {low }}$ cells (Figure S6e, f). To further validate that fatty acid uptake is the major source of LD accumulation in these cells, we suppressed fatty acid uptake by removing extracellular fatty acids using a delipidized serum. Hyperspectral SRS imaging at $\mathrm{C}-\mathrm{H}$ vibrations and phasor analysis showed a significant reduction in the number of LDs when fatty acid availability became limited (Figures 3(h) and 3(i)). Similarly, when fatty acid uptake was suppressed by treating the cells with an inhibitor (BMS309403) targeting fatty acid-binding protein 4 (FABP4), a fatty acid transporter, the number of LDs reduced significantly (Figures 3(h) and 3(i)). Collectively, these data show that fatty acid uptake is the major route for $\mathrm{LD}$ accumulation in $\mathrm{MITF}^{\text {low }} / \mathrm{AXL}^{\text {high }}$ melanoma.

To validate these findings in clinical samples, we analyzed the expression of genes related to lipogenesis and fatty acid uptake in human melanoma samples from TCGA divided into primary and metastatic melanoma (Figure S6g). On average, MITF expression is reduced whereas the AXL expression level increased in the metastatic melanoma group, validating that $\mathrm{MITF}^{\text {low }} / \mathrm{AXL}^{\text {high }}$ melanoma cells represent metastatic melanoma more closely. Importantly, the rate-limiting enzyme in de novo lipogenesis, fatty acid synthase (FASN), showed no difference between primary and metastatic melanomas (Figure S6g), which is consistent with the SRS imaging results for evaluating lipogenesis activity shown in Figures 3(b) and 3(c). However, fatty acid transporters such as CD36 and FABP4 were upregulated in metastatic melanoma compared to primary melanoma (Figure S6g), suggesting increased fatty acid uptake activity. Since metastatic melanoma is a heterogeneous disease, we stratified metastatic melanoma samples into $\mathrm{MITF}^{\text {high }} / \mathrm{AXL}^{\text {low }}$ and MITF $\mathrm{MI}^{\text {low }} / \mathrm{AXL}^{\text {high }}$ groups based on their relative MITF and AXL levels. Within the metastatic melanoma samples, there was a clear difference in the expression of lipid metabolism-related genes based on MITF/AXL status (Figure S6h). The metastatic MITF $^{\text {low }} / \mathrm{AXL}^{\text {high }}$ group showed upregulation of multiple fatty acid transporters and downregulation of genes involved in lipogenesis compared to the MITF ${ }^{\text {low }} / \mathrm{AXL}^{\text {high }}$ group. Together, while lipid de novo synthesis activity is at the same level, fatty acid uptake activity is found to be elevated in human metastatic melanoma.

2.4. Fatty Acid Sapienate Significantly Promotes Cell Migration. To explore the relationship between LD accumulation and melanoma aggressiveness, we classified the melanoma cell lines into two groups based on their LD accumulation level (Table S1). The LD-rich melanoma cells showed significantly higher migration capacity than LDpoor melanoma cells (Figures $4(\mathrm{a})$ and $4(\mathrm{~b})$ ). Since LD accumulation mainly comes from fatty acid uptake (Figure 3), we examined the migration capacity of LD-rich cells after inhibition of fatty acid uptake to deplete LDs. When the LD accumulation was suppressed by depleting extracellular lipids with delipidized serum (Figures 3(f) and $3(\mathrm{~g}))$, the number of migrated cells reduced significantly (Figure 4(c) and Figure S7a). Moreover, when the LD-rich cells were treated with various fatty acid transporter inhibitors, including BMS309403 (FABP inhibitor), sulfosuccinimidyl oleate (CD36 inhibitor), and lipofermata (FATP inhibitor), migration capacity significantly reduced (Figure 4(c) and Figure S7a). These results indicate that LD accumulation, driven by fatty acid uptake, contributes to the enhanced melanoma migration potential.

Next, we asked whether certain fatty acids play a more important role in promoting cell migration than others. We evaluated the migration rescue capability of various fatty acids by supplementing them into the delipidized serum (Figure 4(d) and Figure S7b). Palmitate (PA), palmitoleate (POA), and oleate (OA) did not rescue cell migration in the delipidized serum (Figure 4(d) and Figure S7b). Intriguingly, supplementation of sapienate (SA) and its elongated form, octadecenoate (OCA), rescued cell migration significantly (Figure 4(d) and Figure S7b), indicating an essential role of these fatty acids in cell migration. To validate the presence of sapienate in LD-rich cells, we measured and compared sapienate levels between LD-rich and LD-poor melanomas. Both sapienate (cis-6hexadecenoate) and palmitoleate (cis-9-hexadecenoate) are monounsaturated fatty acids that enhance the unsaturation degree in cells, but the positions of the double bond are different. To specifically measure sapienate, we applied GC/MS to separate and detect sapienate and palmitoleate. Sapienate shows a peak at an earlier retention time than 

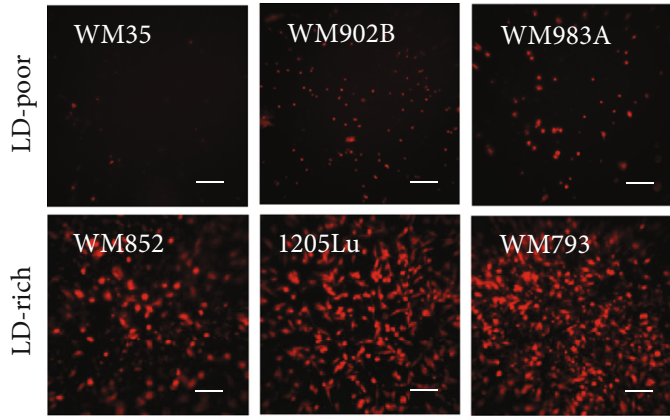
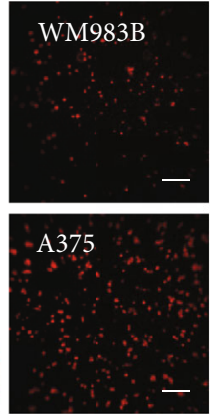

(a)

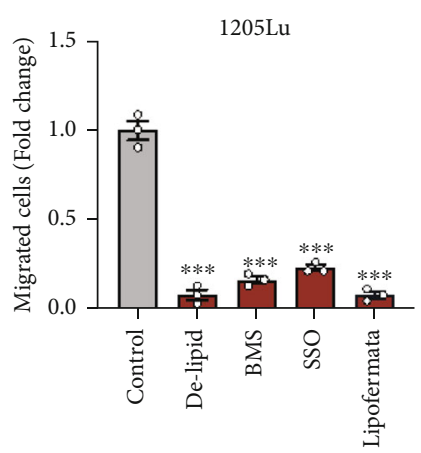

(c)

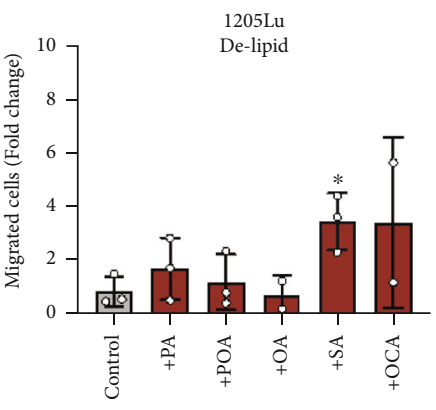

(d)

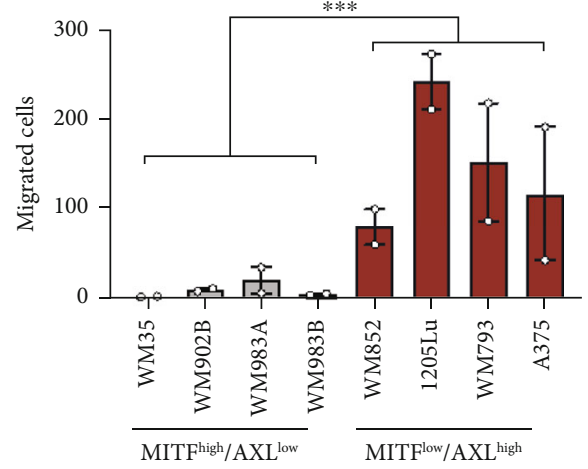

(b)

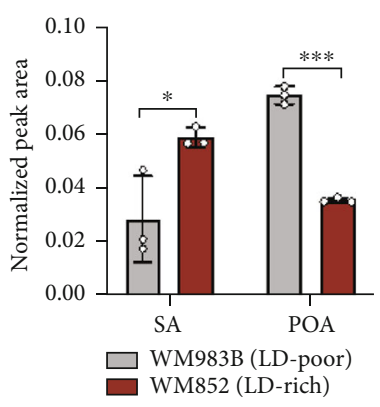

(e)

FIGURE 4: Fatty acid sapienate significantly promotes cell migration. (a) Images and (b) quantification of migrated MITF ${ }^{\text {high }} / \mathrm{AXL}^{\text {low }}$ and $\mathrm{MITF}^{\text {low }} / \mathrm{AXL}^{\text {high }}$ melanoma cells. $n=2$ biological replicates. (c) Quantification of migrated 1205Lu precultured with delipidized medium or pretreated with BMS309403 (BMS, $50 \mu \mathrm{M}, 1$ day), sulfosuccinimidyl oleate (SSO, $50 \mu \mathrm{M}, 1$ day), and lipofermata (10 $\mu \mathrm{M}, 1$ day). Control group was used for normalization. $n=3$ biological replicates. (d) Quantification of migrated 1205Lu precultured with delipidized serum media ( 1 day) and supplemented with ethanol as control and fatty acids $(20 \mu \mathrm{M}, 12$ hours) as indicated. Control group was used for normalization. $n=3$ biological replicates. (e) Quantification of normalized peak areas of sapienate and palmitoleate presented in WM852 and WM983B. $n=3$ biological replicates. Scale bars, $50 \mu \mathrm{m}$. Data represent mean \pm SEM. ${ }^{*} p<0.05$ and ${ }^{* * *} p<0.001$.

palmitoleate (Figure S7c), and this protocol allows us to separate these two fatty acids to obtain the amount of sapienate in the sample. By fatty acid methyl ester (FAME) analysis, we extracted free fatty acids from LD-rich and LD-poor melanoma cells. On the basis of the GC/MS analysis, the LD-rich melanoma is found to contain a much higher amount of sapienate than the LD-poor melanoma (Figure 4(e) and Figure S7d). Interestingly, the palmitoleate content shows the opposite trend, with a higher amount in LD-poor melanoma than in LD-rich melanoma (Figure 4(e)). Together, these results suggest that sapienate is an essential fatty acid in LD-rich melanoma and has an important functional role in enhancing cell migration.

\subsection{Inhibition of FADS2 Suppresses Human Melanoma} Migration In Vitro and Metastasis In Vivo. The opposite trends of sapienate and palmitoleate levels in LD-poor and LD-rich melanoma (Figure 4(e)) triggered us to investigate the role of sapienate metabolism in melanoma aggressive- ness. Sapienate is synthesized from palmitate by FADS2, while palmitoleate is synthesized from palmitate by SCD (Figure 5(a)). A recent study indicates that these two desaturases can be complementary to each other to support cancer progression [26]. Importantly, our TCGA database analysis indicates that while SCD is expressed at similar levels, FADS2 expression is significantly upregulated in metastatic melanoma compared to primary melanoma (Figure S8a). To further evaluate the importance of FADS2mediated fatty acid desaturation in melanoma metastasis, we tested the impact of FADS2 inhibition on cell migration (Figures 5(b) and 5(c)). FADS2 was inhibited by an inhibitor, SC26196, or by shRNA (Figure S8b). After inhibition of FADS2 by SC26196, cell migration was suppressed 1.5 -fold compared to the control group (Figure 5(b)). As additional evidence, FADS2 knockdown by shRNA resulted in reduced cell migration by up to 2 -fold, depending on the degree of knockdown efficiency (Figure 5(c) and Figure S8b). Importantly, sapienate supplementation rescued the 


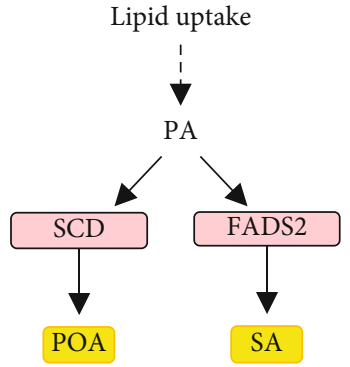

(a)
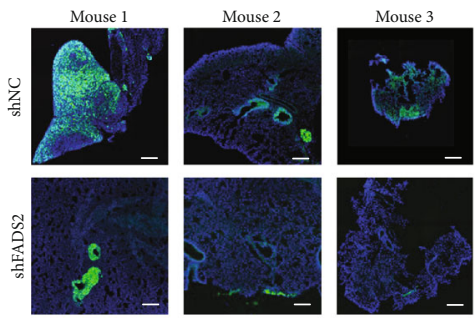

(d)

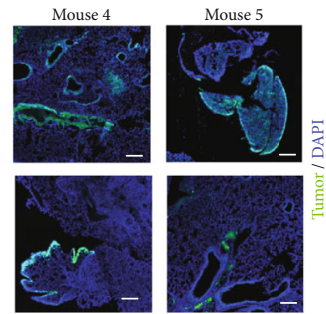

(b)

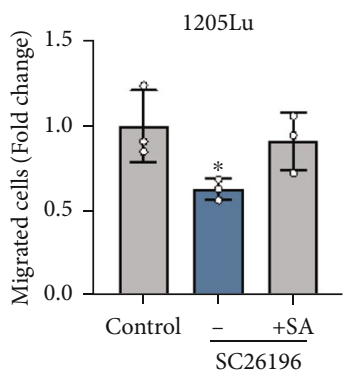

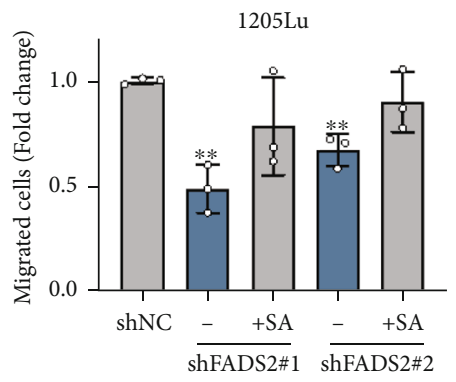

(c)

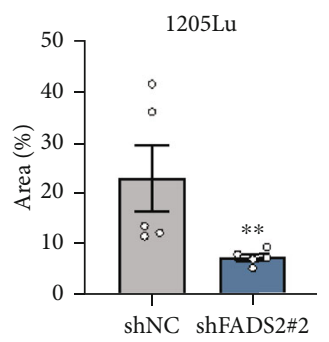

(e)

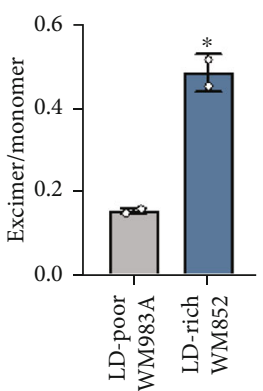

(f)

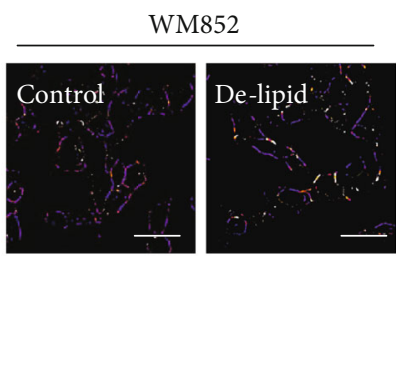

(g)

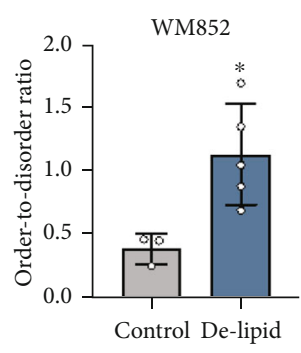

(h)

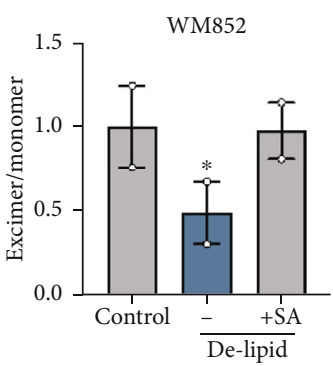

(i)

FIGURE 5: Inhibition of FADS2 suppresses melanoma migration and metastasis through modulation of membrane fluidity. (a) A schematic of two major fatty acid desaturases and their products. (b) Quantification of migrated 1205Lu treated with DMSO as a control, with SC26196 ( $50 \mu \mathrm{M}, 2$ days), and with SC26196 with supplement of sapienate $(20 \mu \mathrm{M}, 1$ day during SC26196 treatment). Control group was used for normalization. $n=3$ biological replicates. (c) Quantification of migrated 1205Lu stabling expressing control shRNA (1205Lu-shNC) or FADS2 shRNAs (1205Lu-shFADS2 \#1 and \#2). 1205Lu-expressing FADS2 shRNAs were cultured in sapienate supplemented medium $(20 \mu \mathrm{M}, 1$ day) for testing the rescue capability. $n=3$ biological replicates. (d) Fluorescence images of lung tissues from mice 46 days after injected with 1205Lu-shNC or 1205Lu-shFADS2 \#1 expressing GFP into the tail vein. Scale bars: $200 \mu \mathrm{m}$. (e) Quantification of percentage area that tumors occupy in lung sections. Data represent mean \pm SEM. $n=5$ mice per group. (f) The ratio between excimer and monomer of membrane fluidity probe (pyrenedecanoic acid, PDA) in WM983A and WM852. $n=2$ biological replicates. (g) Ratiometric images of membrane fluidity probe (di-4-ANESPPDHQ) in WM852 cultured in a normal medium as control and delipidized serum medium. Scale bars: $50 \mu \mathrm{m}$. (h) Quantification of order-to-disorder ratio obtained from (g). $n=3-5$ fields of view. (i) The ratio between excimer and monomer of PDA in WM852 cultured with a normal medium as control, with delipidized serum medium, and with delipidized serum medium with sapienate supplement ( $50 \mu \mathrm{M}, 1$ day). $n=2$ biological replicates. Data represent mean \pm SEM. ${ }^{*} p<0.05$ and ${ }^{* *} p<0.01$.

migration capacity of the cells with either FADS2 inhibition methods (Figures 5(b) and 5(c)), indicating that sapienate has a functional role in promoting melanoma cell migration. Furthermore, FADS2 knockdown suppressed cell invasion, another important property of cancer metastasis, by 2-fold (Figure S8c, d). To evaluate the role of FADS2 in metastasis, we used a mouse tail-vein model. Human melanoma cells stably expressing control shRNA or FADS2 shRNA were intravenously injected, and the amount of lung metastases was compared (Figure 5(d)). Mice injected with melanoma cells containing FADS2 knockdown developed fewer lung metastases compared to the mice injected with control melanoma cells (Figure 5(d)). FADS2 knockdown resulted in a $68 \%$ reduction in the fractional area of lung metastases 
compared to the control group (Figure 5(e)). When the other fatty acid desaturase, SCD1, was inhibited, the migration capacity of $\mathrm{MITF}^{\text {low }} / \mathrm{AXL}^{\text {high }}$ melanoma cells was not significantly changed (Figure S8e, f), suggesting that FADS2 is a specific target for suppressing melanoma metastasis. Together, these results indicate that FADS2 is a potential therapeutic target for suppressing melanoma metastasis.

\subsection{Melanoma Does Not Depend on Fatty Acid Oxidation for} Energy Production, Proliferation, or Migration. One major function of fatty acids is providing energy through $\beta$-oxidation [27]. To investigate whether elevated fatty acid uptake in LD-rich melanoma cells supports fatty acid oxidation, we performed extracellular flux analyses using a Seahorse XFe96 analyzer on melanoma cells treated with or without $4 \mu \mathrm{M}$ etomoxir to inhibit mitochondrial fatty acid oxidation (Figure S9a, b). There were no differences in total ATP generation between etomoxir-treated cells and control cells (Figure S9a). In more detail, we analyzed the energy metabolic status of the cells based on mitochondrial and glycolytic ATP generation (Figure S9b). There were no differences in mitochondrial or glycolytic ATP generation between etomoxir-treated cells and control cells; thus, no metabolic shift was observed. Consistent with this finding, the on-target $5 \mu \mathrm{M}$ etomoxir dose had no effect on LD-rich melanoma cell proliferation while the off-target $200 \mu \mathrm{M}$ etomoxir dose slightly reduced cell proliferation (Figure S9c, d). Although high doses of etomoxir are used ubiquitously, recent research shows that higher doses $(>10 \mu \mathrm{M})$ have off-target effects leading to oxidative phosphorylation suppression through Complex I inhibition, which is erroneously interpreted as a reduction in fatty acid oxidation $[28,29]$. Because of this, caution must be taken when interpreting data using high concentrations of etomoxir to inhibit fatty acid oxidation. Furthermore, LD-rich melanoma cells treated with the ontarget $5 \mu \mathrm{M}$ dose showed no significant change in the cell migration capacity compared to control cells (Figure S9e, f ). Collectively, these results indicate that LDs in metastatic melanoma are not used for fatty acid oxidation in normal, nonstarved conditions.

2.7. Sapienate Modulates Membrane Fluidity. Since unsaturated fatty acids are known to increase membrane fluidity and increased membrane fluidity facilitates cell migration and cancer metastasis [30], we hypothesize that sapienate and its downstream metabolites modulate membrane fluidity. LD-rich melanoma cells show higher membrane fluidity than LD-poor melanoma cells (Figure 5(f)). Furthermore, when the fatty acid uptake was suppressed by culturing cells in delipidized serum, the membrane fluidity was reduced significantly by 2 -fold (Figures $5(\mathrm{~g})$ and $5(\mathrm{~h})$ ). Importantly, sapienate supplemented into the delipidized serum was sufficient to rescue this effect, restoring membrane fluidity to a level similar to the control group (Figure 5(i)). These results suggest that sapienate plays a role in regulating membrane composition and dynamics, which potentially promotes melanoma cell migration in vitro and tumor metastasis in vivo.

\section{Discussion}

Altered lipid metabolism is being increasingly recognized in aggressive cancers [31]. Cancer cells increase their reliance on de novo biosynthesis or exogenous fatty acid uptake. These lipid metabolic features provide biomolecules to sustain rapid proliferation and, at the same time, serve as an energy source during metabolic stress [27]. Among the many aspects of lipid metabolism, fatty acid uptake is shown to be elevated in cancers with high metastatic potential [32, 33]. From this point of view, the metabolic microenvironment of tumors, such as adjacent adipocytes, has been shown to supply lipids and fatty acids to support cancer progression and promote metastasis $[17,34,35]$. In addition to lipid degradation to promote cancer survival [36], fatty acids provide energy through fatty acid oxidation to enhance metastasis $[37,38]$. One particular lipid species that actively contributes to cancer progression is cholesterol [39]. Increased cholesterol biosynthesis and uptake are elevated in aggressive cancers, and the mevalonate pathway, part of cholesterol synthesis, interacts closely with oncogenic pathways $[40,41]$. Accumulation of $\mathrm{CE}$ in LDs is found in multiple types of cancer [39], which serves as a reservoir of excess cholesterol to maintain cholesterol synthesis and uptake. These studies highlight the importance of lipid metabolism during cancer progression.

Despite various efforts to understand lipid metabolic reprogramming in cancer, the alterations in composition, intracellular distribution, and dynamics of lipids remain understudied. Lipids are highly dynamic and complicated species with functional roles dependent on their distribution. For example, lipid composition changes the physical properties of the membrane, such as fluidity, protein localization and activity, cell adhesion, and migration [30]. LDs are specialized storage organelles receiving attention because of their multiple functions such as serving as an energy source [42], supporting membrane biosynthesis [43], protecting cells from lipotoxicity [44] and oxidative stress [45], maintaining ER homeostasis [46], and interactions with other organelles [47]. Analyzing the composition of LDs and their spatiotemporal dynamics in cancer may suggest connections between oncogenic events and metabolic reprogramming for the development of new therapeutic targets.

High-speed, high-resolution chemical imaging enabled by coherent Raman scattering microscopy offers an effective tool to understand the altered metabolism inside cancer cells [48]. By SRS imaging and Raman spectroscopic analysis of single LDs in human prostate cancer patient tissues and cells, accumulation of $\mathrm{CE}$ in aggressive cancer was discovered, and cholesterol esterification was demonstrated as a therapeutic target of metastatic prostate cancer [49]. Using single-cell hyperspectral SRS imaging of ovarian cancer, unsaturated lipid was identified as the metabolic signature of cancer stem cells, and inhibition of lipid desaturation can effectively eliminate cancer stem cells [50]. Enabled by the high-speed spectral acquisition by the multiplexed SRS imaging scheme, the high-content analysis using SRS imaging cytometry unveiled a metabolic response to stress in cancer cells, indicating LD-rich protrusions with high 
lipolysis and fatty acid oxidation activities to support cancer survival under starvation and chemotherapy [51]. Collectively, these studies demonstrate the capability of coherent Raman scattering microscopy in understanding metabolic alterations in cancer with subcellular resolution and temporal dynamics.

An intriguing question is why metastatic melanoma favors fatty acid uptake over de novo lipogenesis. One possible reason is that melanoma is developed in a lipid-rich local environment [17, 34]. Upregulation of several fatty acid transporter genes, such as FABP4 [33, 34] and CD36 [32, $52]$, has been reported in metastatic cancer. It was found that stress such as hypoxia upregulates FABP4 expression [33]. Following enhanced fatty acid uptake, fatty acid oxidation is found to play an important function in cancers. For example, fatty acid oxidation helps cancer cells to survive under metabolic stress [51]. In melanoma, fatty acid oxidationdependent drug resistance has been reported [53]. Inhibition of fatty acid oxidation suppresses metastasis to local lymph nodes [37], indicating the importance of fatty acids in promoting local metastasis in melanoma. Intriguingly, bloodborne metastasis to the lungs was not suppressed by fatty acid oxidation inhibition [37], suggesting that other functions of fatty acids exist that promote melanoma metastasis beyond local lymph nodes. In this study, we identified another important function of fatty acids in the cell, which is through the regulation of membrane fluidity and posttranslational modifications of proteins.

A recent independent study by $\mathrm{Du}$ et al. showed increased de novo fatty acid synthesis in one of the primary melanoma cell lines that belongs to the differentiated type [54]. In their work, while the undifferentiated type showed much lower de novo fatty acid synthesis, it contained LDs with a much higher unsaturation level. Here, we identified increased fatty acid uptake activities that contribute to LD accumulation in multiple undifferentiated $\mathrm{MITF}^{\text {low }} / \mathrm{AXL}^{\text {high }}$ melanoma cell lines. Fatty acid uptake as the major source of LD accumulation, but not de novo lipogenesis, is further supported by the results showing no difference of the LD amount in metastatic melanoma after culturing in glucosefree medium (Figures 3(d) and 3(e)). It is exciting that both studies identified common metabolic phenotypes of metastatic melanoma, i.e., increased cholesteryl ester and unsaturation degree of lipids.

There are two major fatty acid monodesaturation pathways: SCD-mediated, generating palmitoleate, and FADS2mediated, generating sapienate. SCD is essential for cancer cell survival, and its anticancer potential has been reported in multiple cancers [55]. However, not all cancer cells are responsive to SCD inhibition [31], and a recent study demonstrated FADS2 as an alternative desaturation pathway in SCD-independent cancer cells [26]. Unlike other fatty acids, sapienate is only found in humans and is a major component of human sebum [56]. Intriguingly, a recent study indicated that SCD expression is positively regulated by MITF [57]. Therefore, one potential mechanism of increased dependency on FADS2-mediated fatty acid desaturation in MITF $^{\text {low }} / \mathrm{AXL}^{\text {high }}$ LD-rich melanoma cell lines could be a compensatory mechanism due to reduced MITF-regulated
SCD. At the same time, a recent report revealed that FADS2-mediated sapienate metabolism is regulated by the mTOR-SREBP1/2 axis [58], providing another possibility for the regulatory mechanism of FADS2 in melanoma. Importantly, as the high migration and invasion capacities presented in the melanoma can be suppressed by FADS2 inhibition in vitro and in vivo, inhibition of FADS2mediated fatty acid desaturation offers a therapeutic opportunity to treat metastatic melanoma.

In addition to serving as a $\Delta 6$ desaturase to generate sapienate from palmitate, FADS2 mediates desaturation to generate polyunsaturated fatty acids. In metastatic melanoma, this sapienate synthetic enzyme is a major contributor promoting cell migration. This function is supported by the capacity of sapienate to rescue cell migration capacity in the delipidized serum condition. Further, the higher amount of sapienate found in LD-rich melanoma compared to LD-poor melanoma suggests elevated sapienate synthesis in metastatic melanoma. Mechanistically, unsaturated lipids on the plasma membrane are known to promote cell mobility [30]. In line with this observation, the membrane fluidity is higher in LD-rich melanoma compared to LD-poor melanoma, and supplementation of sapienate increases membrane fluidity. Intriguingly, the supplementation of palmitate in delipidized serum slightly rescued migration capacity. It is likely that high FADS2 activity in MITF ${ }^{\text {lo- }}$ w/AXL high melanoma cells converted the palmitate into sapienate, thus increasing the membrane fluidity. It would be important to test this hypothesis to unveil the functional roles of specific fatty acids in cancer cells. We note that unsaturated fatty acids in general would enhance the membrane fluidity. Therefore, this mechanism may not be specific to sapienate alone. Nevertheless, these results collectively indicate an essential function of sapienate in cancer migration.

In parallel to the reprogramming of fatty acid metabolism in metastatic melanoma, cholesterol metabolism also plays an essential role in promoting cell migration. A significant amount of CE in LDs of metastatic melanoma suggests a rewiring of cholesterol metabolism. CE is synthesized from cholesterol and fatty acids by SOAT protein (Figure S10a). With the increasing number of reports regarding the upregulation of SOAT-mediated cholesterol esterification in cancers [39], it is essential to understand the physiological meaning and function of $\mathrm{CE}$ accumulation in metastatic disease. Our results demonstrate that SOAT inhibition effectively suppresses melanoma migration (Figure S10b, c), indicating that cholesterol esterification is another potential target for metastatic melanoma. Based on the reduced LD accumulation observed in SOAT-inhibited melanoma (Figure S10d), it is likely that cholesterol esterification is connected to fatty acid uptake, storage, and utilization. Fatty acid is known to serve as a substrate for protein modification such as palmitoylation to regulate protein localization and activities. For example, Wnt family proteins require palmitoylation to be transported to the plasma membrane for secretion [59]. Indeed, when cholesterol esterification is inhibited by avasimibe, $\beta$ catenin is sequestered on the plasma membrane (Figure S10e), indicating inactivation of the Wnt/ $\beta$-catenin 
pathway. Furthermore, the amount of membrane-bound Wnt5a reduced significantly by 3 -fold when cholesterol esterification was inhibited by avasimibe (Figure S10f). A similar Wnt5a distribution was found in melanoma cells cultured with delipidized serum (Figure S10g), which limits fatty acid uptake. Although cholesterol itself can modify signaling molecules such as the hedgehog protein [60], we did not observe inactivation of the hedgehog pathway when cholesterol esterification is inhibited (Figure S10h). Together, these results indicate that the cholesterol metabolism plays an important role in melanoma metastasis, possibly mediated by a change of fatty acid availability for protein modification (Figure S10i). As Wnt5a is a robust marker of aggressive and metastatic melanoma [61], our finding of modulation of Wnt5a modification by reprogrammed lipid metabolism provides an essential mechanism of linking metabolism with signaling pathways.

Finally, the metabolic reprogramming from pigmentation to lipid accumulation identified in this study serves as a molecular signature for metastatic melanoma diagnosis. It was found that more eumelanin than pheomelanin is present in malignant melanoma compared to dysplastic nevi [6]. Using pump-probe imaging, the distribution of these two types of melanin was studied in human tissues, which provides a label-free imaging approach for melanoma diagnosis [8]. More recently, chemical analysis of melanin with its spatial distribution using pump-probe microscopy further demonstrated the potential of predicting the metastatic capability of melanoma [9]. Despite these advances, loss of MITF found in melanomas with an invasive phenotype [10] suggests loss of pigmentation, indicating a need to identify new molecular markers for detecting aggressive and invasive melanomas. In this study, by the integration of label-free SRS imaging of lipids and pump-probe imaging of pigments, we identified a metabolic reprogramming from pigmentation to lipid accumulation in metastatic melanoma. Such a multimodal imaging platform can potentially be used to diagnose primary melanoma and metastatic lesions in human tissue samples. Furthermore, with the continued development of a handheld SRS imaging device [62], in vivo SRS and pump-probe imaging promises a noninvasive approach for the clinical evaluation of melanoma.

\section{Materials and Methods}

4.1. Cell Lines and Cell Culture. Human melanoma cell lines (WM35, WM902B, WM983A, WM983B, 1205Lu, WM793, and A375) harboring the BRAF V600E mutation and human melanoma cell line (WM852) with wildtype BRAF were obtained from Dr. Meenard Herlyn (The Wistar Institute). Cells were grown in Dulbecco's Modified Eagle's Medium (DMEM, Invitrogen) media supplemented with fetal bovine serum (FBS) (10\%), L-glutamine $(2 \mathrm{mM})$, penicillin (1\%), and streptomycin (1\%). Human primary melanocytes (Life Technologies) were grown in Medium 254 (Gibco) with human melanocyte growth supplements (Gibco). Cell lines were incubated at $37^{\circ} \mathrm{C}$ in $5 \% \mathrm{CO}_{2}$.
4.2. Chemicals. FBS was purchased from Life Technologies. Delipidized serum was purchased from Gemini Bio. CAY10566, BMS309403, sulfosuccinimidyl oleate, lipofermata, and etomoxir were purchased from Cayman Chemical. SC26196 was purchased from Santa Cruz. Avasimibe was purchased from Selleckchem. Glucose- $\mathrm{D}_{7}$, palmitic acid- $\mathrm{D}_{31}$, and oleic acid- $\mathrm{D}_{34}$ were purchased from Cambridge Isotope Laboratories. Palmitate, palmitoleate, oleate, cholesteryl oleate, and glyceryl trioleate were purchased from SigmaAldrich. Sapienate was purchased from Matreya. Octadecenoate $(8(Z)$-octadecenoic acid) was purchased from Larodan.

4.3. Time-Domain Multimodal SRS/Pump-Probe Imaging. SRS/pump-probe imaging was performed on a femtosecond SRS microscope. An ultrafast laser system with dual output at $80 \mathrm{MHz}$ (InSight DeepSee, Spectra-Physics) provided pump and Stokes beams. In the SRS imaging scheme, $802 \mathrm{~nm}$ and $1045 \mathrm{~nm}$ beams serve as pump and Stokes beams, respectively, to be resonant with the C-H stretching vibration at $2899 \mathrm{~cm}^{-1}$. For the pump-probe imaging scheme, $802 \mathrm{~nm}$ and $1045 \mathrm{~nm}$ beams serve as probe and pump beams, respectively. For off-resonance imaging of the $\mathrm{C}-\mathrm{H}$ stretching vibration, the pump beam was tuned to $845 \mathrm{~nm}$, which corresponds to $2265 \mathrm{~cm}^{-1}$. In this imaging scheme, the pump-probe signal, generated by $1045 \mathrm{~nm}$ pump and $845 \mathrm{~nm}$ probe, becomes dominant because no endogenous Raman active biomolecules are presented in the samples. $1045 \mathrm{~nm}$ beam was modulated by an acoustooptic modulator (AOM, 1205-C, Isomet) at 2.2 MHz. Both beams were linearly polarized. A motorized translation stage was employed to scan the temporal delay between the two beams. Two beams were then sent into a home-built laserscanning microscope. A 60x water immersion objective lens (NA = 1.2, UPlanApo/IR, Olympus) was used to focus the light into the sample, and an oil condenser $(\mathrm{NA}=1.4$, UAAC, Olympus) was used to collect the signal. The stimulated Raman loss and pump-probe signals were detected by a photodiode, which was extracted with a digital lock-in amplifier (Zurich Instrument). The power of the tunable beam $(802 \mathrm{~nm}$ and $845 \mathrm{~nm})$ and the power of the $1045 \mathrm{~nm}$ beam at the specimen were maintained at $\sim 10 \mathrm{~mW}$ and 5 to $25 \mathrm{~mW}$, respectively. The images were acquired at $10 \mu \mathrm{s}$ pixel dwell time. No cell or tissue damage was observed during the imaging procedure.

4.4. Phasor Analysis for LD and Pigment Quantification. LDs and pigments were analyzed by phasor analysis using the ImageJ plugin [23]. The segmentation of LDs and pigments in the phasor space is shown in Figure S2. After the segmentation, the "Threshold" function was used to select droplets in the cells. The "Analyze Particles" function was then used to quantify the area fractions of droplets in the whole image area, then normalized to the cell number counted from the same image.

4.5. In Silico Analysis of Melanoma Using TCGA Datasets. The transcriptome dataset of melanoma tissues (TCGASKCM) obtained from TCGA was downloaded in Apr 2019 using the UCSC Xena browser. The patients were 
assigned to $\mathrm{MITF}^{\text {high }} / \mathrm{AXL}^{\text {low }}$ and $\mathrm{MITF}^{\text {low }} / \mathrm{AXL}^{\text {high }}$ groups based on the median expression level. Differentially expressed genes and analyses via Gene Set Enrichment Analysis were generated using NetworkAnalyst 3.0 [63]. Further enrichment analyses were performed in the included genes (FC $>1.2$, adjusted $p<0.05$ ) using Metascape [64].

4.6. Human Tissue Sample Preparation. Deidentified frozen specimens of human primary and metastatic melanoma tissues were purchased from IU Simon Comprehensive Cancer Center (Lafayette, IN). Informed written consent from all participants or next of kin was obtained prior to the research by the IU Simon Comprehensive Cancer Center. The study is approved by the Boston University Institutional Review Board (2256). The tissue samples were sliced using a cryostat at 10 to $20 \mu \mathrm{m}$ thickness. The time-domain multimodal SRS/pump-probe imaging was performed on these tissue slices without any processing or labeling.

4.7. Confocal Raman Spectral Measurement. Raman spectral analysis from individual LDs was performed using a commercial confocal Raman microscope (LabRAM HR Evolution, Horiba) at room temperature. A $15 \mathrm{~mW}$ (after the objective), $532 \mathrm{~nm}$ diode laser was used to excite the sample through a 40x water immersion objective (Apo LWD, 1.15 N.A., Nikon). The total data acquisition time was $60 \mathrm{~s}$ using the LabSpec 6 software. For each group, at least 10 spectra from individual LDs in different locations or cells were obtained. To analyze the spectrum, the background was removed manually based on the glass background profile, and peak intensity was measured using OriginPro. The calibration curve for CE percentage in LD was generated by measuring $\mathrm{CE} /$ triacylglyceride emulsion at various percentages and linearly correlate it with the $704 \mathrm{~cm}^{-1}$ peak (cholesterol rings) normalized with the $1445 \mathrm{~cm}^{-1}$ peak $\left(\mathrm{CH}_{2}\right.$ bending vibration). The unsaturation degree of $\mathrm{LD}$ is determined by the peak intensity at $1654 \mathrm{~cm}^{-1}(\mathrm{C}=\mathrm{C}$ stretching vibration) normalized with the $1445 \mathrm{~cm}^{-1}$ peak.

4.8. Metabolite Extraction and Measurement by LC/MS and GC/MS. The cells were extracted using a modified BlighDyer extraction [65]. To each sample, $0.4 \mathrm{~mL}$ of ice-cold $65 \%$ methanol was added and vortexed for $1 \mathrm{~min}$. The samples were placed on ice, spiked with $1 \mu \mathrm{g}$ of $\mathrm{C} 17: 0$ margaric acid as internal standard, then pulse vortexed to mix. Next, $0.25 \mathrm{~mL}$ of chloroform was added and the sample vortexed for an additional 5 minutes. The samples were centrifuged at $13,500 \mathrm{rpm}$ for 5 minutes at $4^{\circ} \mathrm{C}$. The bottom layer was removed for fatty acid analysis. The samples were dried using a rotary evaporation device at room temperature for 2 hours. The samples were then derivatized for GC/MS analysis. Each sample received $0.5 \mathrm{~mL}$ of $14 \%$ boron trifluoride solution $\left(\mathrm{BF}_{3}\right)$ (Sigma-Aldrich \#B1252) and reacted for 30 minutes at $60^{\circ} \mathrm{C}$. Then, $0.25 \mathrm{~mL}$ of water and $1 \mathrm{~mL}$ of hexane were added. The samples were mixed, then dried with approximately $0.2 \mathrm{~g}$ of anhydrous sodium sulfate. The hexane layer was then collected and dried using a stream of nitrogen. The final derivatized sample was reconstituted in $0.1 \mathrm{~mL}$ of hexane for GC/MS analysis.
TABLE 1: SIM table for mass spectrometry analysis.

\begin{tabular}{lccc}
\hline Name & RT & Window $(\min )$ & SIM mass \\
\hline Palmitic $(16: 0)$ acid & 11.9 & 1 & 227 \\
Sapienic $(16: 1 \Delta 6)$ acid & 12.4 & 1 & 236 \\
Palmitoleic $(16: 1 \Delta 9)$ acid & 12.6 & 1 & 236 \\
Margaric $(17: 0)$ acid-ISTD & 13.2 & 1 & 241 \\
\hline
\end{tabular}

A Thermo Fisher TriPlus RSH auto sampler and Trace 1310 gas chromatography (GC) system coupled to a Thermo Fisher TSQ 8000 mass spectrometer (MS) was used to analyze FAME composition in each sample (Thermo Fisher Scientific, Waltham, MA). An Agilent Select FAME GC column $(50 \mathrm{~m} \times 0.25 \mathrm{~mm}$, film thickness $0.2 \mu \mathrm{m})$ was used for the analysis (Agilent Technologies, Santa Clara, CA). The GC carrier gas was helium with a linear flow rate of $1.0 \mathrm{~mL} \mathrm{~min}^{-1}$. The programmed GC temperature gradient was as follows: time 0 minutes, $80^{\circ} \mathrm{C}$, ramped to $175^{\circ} \mathrm{C}$ at a rate of $13^{\circ} \mathrm{C} \mathrm{min}^{-1}$ with a 5 -minute hold, then ramped to $245^{\circ} \mathrm{C}$ at a rate of $4^{\circ} \mathrm{C} \mathrm{min}^{-1}$ with a 2 -minute hold. The total run time was 38.3 minutes. The $\mathrm{GC}$ inlet was set to $250^{\circ} \mathrm{C}$, and samples were injected in the split-less mode. The MS transfer line was set to $250^{\circ} \mathrm{C}$, and the MS ion source was set to $250^{\circ} \mathrm{C}$. MS data were collected in the selected ion monitoring (SIM) mode according to Table 1. All data were analyzed with Thermo Fisher Chromeleon (Version 7.2.9) software. A standard mixture of 37 FAME (Supelco, Sigma-Aldrich), sapienate (Larodan), and palmitoleate (Sigma-Aldrich) was used to confirm spectra and column retention times.

4.9. Isotope Metabolite Labeling Experiments. For tracking de novo lipogenesis, cells are cultured with glucose- $\mathrm{D}_{7}\left(4.68 \mathrm{~g} \mathrm{~L}^{-}\right.$

${ }^{1}$ ) in the glucose-free complete medium for 48 hours. For tracking fatty acid uptake, $100 \mu \mathrm{M}$ of palmitic acid- $\mathrm{D}_{31}$ or oleic acid- $\mathrm{D}_{34}$ was supplemented in the complete medium and cultured for 6 hours. The pump laser was tuned to $855 \mathrm{~nm}$ to perform SRS imaging in the C-D region at $2127 \mathrm{~cm}^{-1}$. Pump and Stokes powers at the specimen were maintained at $\sim 10 \mathrm{~mW}$ and $50 \mathrm{~mW}$, respectively. To quantify the SRS intensity at the C-D region from LDs, the "Threshold" function in ImageJ was used to select LDs in the cells. Then, the total intensity of LDs was obtained after particle analysis and normalized by the number of cells in the corresponding image.

4.10. Hyperspectral SRS Imaging and Phasor Analysis. Hyperspectral SRS imaging was performed by a spectral focusing approach. Both beams were chirped by three $12.7 \mathrm{~cm}$ long SF57 glass rods with the Stokes beam chirped by additional glass rod to match the pulse durations of the both beams. The Raman shift was calibrated using DMSO. The images were acquired at $10 \mu$ s pixel dwell time. No cell damage was observed during the imaging procedure. LDs were then analyzed by phasor analysis using the ImageJ plugin [23]. The cells were segmented into LD, ER, cytoplasm, and nucleus by spectral phasor. 
4.11. Migration/Invasion Assay. 0.2 million cells were seeded in the upper chamber of a 24-well plate insert (Corning ${ }^{\mathrm{TM}}$ Transwell $^{\mathrm{TM}} 8 \mu \mathrm{m}$ Permeable Polycarbonate Membrane Inserts) in serum-free media. For the invasion assay, the insert was precoated with Matrigel (BD Biosciences). DMEM media supplemented with $20 \%$ FBS and $50 \mathrm{ng} \mathrm{mL}^{-1}$ EGF were added to the bottom chamber of the insert. For the treatment group, inhibitors or fatty acids were added to both the upper and bottom chambers. After 4.5 to 8 hours of migration, cells were fixed with either $10 \%$ formalin or $70 \%$ ethanol, and nonmigrated cells were removed with a cotton swab. Migrated cells were stained with $50 \mu \mathrm{g} \mathrm{mL}$ propidium iodide (Invitrogen) for 30 minutes at room temperature and then washed with phosphate-buffered saline 5 to 6 times. 8 to 10 random images were taken for each well using an FV3000 confocal microscope or a Nikon Eclipse E400 microscope. The number of migrated/invaded cells was quantified using ImageJ. Migrated cell count was normalized to the control group and represented as fold change from control unless indicated otherwise.

4.12. Knockdown Strategies. 1205Lu cells were transfected with FADS2 targeting shRNA lentiviral particles (Applied Biological Materials, shFADS2 \#1: CCGGCCACGGCAAG AACTCAAAGA-TCTCGAGATCTTTGAGTTCTTGCC GTGGTTTTTG, shFADS2 \#2: CCGGCCAC CTGTCTGTCTACAGAAACTCGAGTTTCTGTAGACAGACAGGT GGTTTTTG) following the protocol provided by the manufacturer. Scrambled shRNA lentiviral particles (shNC: GTCTCCACGCGCAGTACATTT) were used as a control. Stably transfected cells were selected with $1 \mu \mathrm{g} \mathrm{mL}^{-1}$ puromycin. 1205Lu and WM852 cells were transfected with SOAT1 targeting shRNA lentiviral particles (Santa Cruz, sc-29624-V) following the protocol provided by the manufacturer. Stably transfected cells were selected with $1 \mu \mathrm{g} \mathrm{mL}^{-1}$ puromycin.

4.13. Mouse Model and Fluorescence Imaging of Tissue Sections. All animal procedures were approved by Boston University IACUC (PROTO201800533). The mouse tailvein injection model was used to study the development of metastatic cancer. 6-week-old male homozygous nude (Foxn1nu/Foxn1nu) mice obtained from the Jackson Laboratory were used. 1205Lu melanoma cells stabling expressing GFP (Applied Biological Materials) were transfected with lentivirus carrying negative control shRNA (1205Lu-shNC) or FADS2 targeting shRNA (1205Lu-shFADS2 \#2). The cells were then prepared in sterile PBS in a completely monocellular suspension without clumps at $1 \times 10^{6} \mathrm{~mL}^{-1}$ concentration. $100 \mu \mathrm{L}$ of cell suspension was injected slowly via the lateral tail vein of the anesthetized mouse, after which the bleeding was stopped by applying pressure to the puncture site with a dry piece of gauze. The lung tissues were collected 46 days after tumor inoculation and sliced using a cryostat at 10 to $20 \mu \mathrm{m}$ thickness. Then, the tissues were mounted with the antifade mounting medium with DAPI (Vector Laboratories). The tissues were imaged using an Olympus VS120 Automated Slide Scanning Microscope.
4.14. Immunofluorescence Staining and Imaging. Cells were fixed with $10 \%$ formalin for $15 \mathrm{~min}$ at room temperature. Cells were then incubated with anti-adipophilin (Millipore Sigma, 393A-1, 1:100), anti-FADS2 (Proteintech, 28034-1AP, 1:200), anti-Glil (Abcam, ab49314, 1:200), anti-active $\beta$-catenin (Millipore Sigma, 05-665, 1:200), or anti-Wnt5a (Santa Cruz, sc-365370, 1:200). After incubation with the secondary antibody containing Alexa-Fluor 488, cells were mounted using the antifade mounting medium with DAPI (Vector Laboratories). Then, the cells were imaged under an FV3000 confocal microscope with a 60x oil objective. The fluorescence signal from the antibody was imaged with $488 \mathrm{~nm}$ excitation and $500-600 \mathrm{~nm}$ emission. DAPI signal was imaged with $405 \mathrm{~nm}$ excitation and $430-479 \mathrm{~nm}$ emission.

4.15. Membrane Fluidity Assay. Membrane fluidity was measured using a MarkerGene ${ }^{\mathrm{TM}}$ Membrane Fluidity Kit (Abcam) following the protocol provided by the manufacturer. Briefly, cells were grown on 96-well plates. After 24 to 48 hours, the cells were washed with PBS and incubated with $10 \mu \mathrm{M}$ of pyrenedecanoic acid (PDA) in a perfusion buffer containing $0.08 \%$ pluronic F127 for 20 minutes at $25^{\circ} \mathrm{C}$ in the dark. Then, the unincorporated PDA is removed by washing the cells with serum-free media twice. The incorporated PDA was measured by reading fluorescence signals at both 400 (monomer) and $460 \mathrm{~nm}$ (excimer) with excitation at $360 \mathrm{~nm}$ using a SpectraMax i3x Microplate Detection Platform (Molecular Devices). The membrane fluidity was represented as a ratio between excimer fluorescence and monomer fluorescence, in which a higher ratio indicates higher membrane fluidity.

4.16. Confocal Imaging of Membrane Fluidity. For imaging membrane fluidity, cells were grown on glass coverslips for 48 hours. Cells were stained with di-4-ANEPPDHQ (Thermo Fisher Scientific) following the protocol provided by the manufacturer. Briefly, $5 \mathrm{mM}$ of di-4-ANNEPPDHQ stock solution in DMSO was diluted in serum-free DMEM (final concentration: $5 \mu \mathrm{M}$ ). Cells were incubated with di-4ANNEPPDHQ-containing DMEM for 30 minutes at $37^{\circ} \mathrm{C}$ in the dark. Then, cells were washed with DMEM and imaged using an FV3000 confocal microscope with excitation at $488 \mathrm{~nm}$ and emission at both 530-590 (ordered lipid) and 590-650 nm (disordered lipid). The images were analyzed using ImageJ to generate ratiometric results of ordered-to-disordered lipids.

4.17. Statistical Analysis. Statistical analysis was performed using OriginPro or Prism. For two-sample comparisons, data were first tested for normality. If the data is detectably non-Gaussian, a nonparametric Mann-Whitney $U$ test was performed. Otherwise, a one-tailed Student's $t$-test was performed. The probability of the null hypothesis $p<0.05$ was judged to be statistically significant.

\section{Data Availability}

The data used to support the findings of this study are available from the corresponding authors upon request. 


\section{Disclosure}

Hyeon Jeong Lee's present address is the College of Biomedical Engineering and Instrument Science, Key Laboratory for Biomedical Engineering of Ministry of Education, Zhejiang University, Hangzhou 310027, China. The content is solely the responsibility of the authors and does not necessarily represent the official views of the National Institute of Health.

\section{Conflicts of Interest}

The authors declare that they have no competing interests.

\section{Authors' Contributions}

HJL, JXC, RA and codesigned the experiments. HJL and ZC performed the experiments and data analysis. MC and JGC performed experiments and data analysis related to fatty acid oxidation. MW and MC performed measurements for MITF/AXL expressions on melanoma. HJL, JXC, and ZC cowrote the manuscript. All authors have read and commented on the manuscript. Hyeon Jeong Lee and Zhicong Chen contributed equally to this work.

\section{Acknowledgments}

The authors thank the IUSCC Cancer Center at Indiana University School of Medicine funded by the IU Simon Cancer Center Support Grant P30 CA082709, for the use of the Tissue Procurement and Distribution Core, which provided Frozen Tissue Sample service. The research reported in this publication was supported by the Boston University Micro and Nano Imaging Facility and the Office of the Director, National Institutes of Health, of the National Institutes of Health under award Number S10OD024993. The Seahorse XFe96 Extracellular Flux experiments were supported by the Boston University Analytical Instrumentation Core. The authors acknowledge the use of the facilities of the Bindley Bioscience Center, a core facility of the NIH-funded Indiana Clinical and Translational Sciences Institute. The authors also acknowledge the Animal Science Center for providing technical support for the animal study reported within this paper. The authors thank Kai-Chih Huang, Peng Lin, Hongli Ni, and Haonan Lin for their help on SRS imaging and pump-probe imaging; thank Junjie Li and Yuying Tan for their helpful discussions; and thank Amber S. Jannasch and Bruce Cooper from Purdue Metabolomics Facility for their help on mass spectrometry measurements. This research is supported by NIH grants R33 CA223581 and R35 GM136223 to JXC.

\section{Supplementary Materials}

Figure S1: imaging LD and pigments in human melanoma cells grouped by MITF and AXL expressions. Table S1: summary of human melanoma cell lines used in the study. Figure S2: pigments and LDs can be separated by timedomain multimodal SRS/pump-probe imaging and phasor analysis. Figure S3: functional enrichment network analysis for human melanoma grouped based on MITF and AXL. Figure S4: hematoxylin and eosin (H\&E) staining of adjacent slices used for multimodal SRS imaging in Figure 1(e). Figure S5. LDs in $\mathrm{MITF}^{\text {low }} / \mathrm{AXL}^{\text {high }}$ melanoma cells contain unsaturated fatty acids and CE. Figure S6. MITF ${ }^{\text {low }} / \mathrm{AXL}^{\text {high }}$ melanoma shows higher oleate uptake activity compared to $\mathrm{MITF}^{\text {high }} / \mathrm{AXL}^{\text {low }}$ melanoma. Figure S7: fatty acid sapienate significantly promotes cell migration. Figure S8: inhibition of FADS2 suppresses melanoma invasion. Figure S9: fatty acid $\beta$-oxidation is not the major source of energy in melanoma. Figure S10: inhibition of cholesterol esterification suppresses melanoma migration via regulation of $\mathrm{Wnt} / \beta$ catenin pathway. Supplementary methods Table S2: GSEA of TCGA-SKCM cohort (MITF ${ }^{\text {high }} / \mathrm{AXL}^{\text {low }}$ vs. $\mathrm{MITF}^{\text {lo- }}$ ${ }^{\mathrm{w}} / \mathrm{AXL}^{\text {high }}$ ) with KEGG, Reactome, and GO-BP subset. Table S3: GO subset enrichment analysis of DEGs between $\mathrm{MITF}^{\text {high }} / \mathrm{AXL}^{\text {low }}$ and $\mathrm{MITF}^{\text {low }} / \mathrm{AXL}^{\text {high }}$ patients. (Supplementary Materials)

\section{References}

[1] S. J. Welsh, H. Rizos, R. A. Scolyer, and G. V. Long, "Resistance to combination BRAF and MEK inhibition in metastatic melanoma: where to next?," European Journal of Cancer, vol. 62, pp. 76-85, 2016.

[2] S. A. Weiss, J. D. Wolchok, and M. Sznol, "Immunotherapy of melanoma: facts and hopes," Clinical Cancer Research, vol. 25, no. 17, pp. 5191-5201, 2019.

[3] W. R. Shaikh, S. W. Dusza, M. A. Weinstock, S. A. Oliveria, A. C. Geller, and A. C. Halpern, "Melanoma thickness and survival trends in the United States, 1989 to 2009," Journal of the National Cancer Institute, vol. 108, article djv294, 2016.

[4] D. C. Whiteman, P. D. Baade, and C. M. Olsen, "More People Die from Thin Melanomas $(\leq 1 \mathrm{~mm})$ than from Thick Melanomas (\&gt;4 mm) in Queensland, Australia," Journal of Investigative Dermatology, vol. 135, no. 4, pp. 11901193, 2015.

[5] A. M. Glazer, R. R. Winkelmann, A. S. Farberg, and D. S. Rigel, "Analysis of trends in US melanoma incidence and mortality," JAMA Dermatology, vol. 153, no. 2, pp. 225-226, 2017.

[6] R. Marchesini, A. Bono, and M. Carrara, "In vivo characterization of melanin in melanocytic lesions: spectroscopic study on 1671 pigmented skin lesions," Journal of Biomedical Optics, vol. 14, no. 1, article 014027, 2009.

[7] T. H. Nasti and L. Timares, "MC1R, eumelanin and pheomelanin: their role in determining the susceptibility to skin cancer," Photochemistry and Photobiology, vol. 91, no. 1, pp. 188-200, 2015.

[8] T. E. Matthews, I. R. Piletic, M. A. Selim, M. J. Simpson, and W. S. Warren, "Pump-probe imaging differentiates melanoma from melanocytic nevi," Science Translational Medicine, vol. 3, no. 71, article 71ra15, 2011.

[9] F. E. Robles, S. Deb, J. W. Wilson et al., "Pump-probe imaging of pigmented cutaneous melanoma primary lesions gives insight into metastatic potential," Biomedical Optics Express, vol. 6, no. 9, pp. 3631-3645, 2015.

[10] S. Carreira, J. Goodall, L. Denat et al., "Mitf regulation of Dia1 controls melanoma proliferation and invasiveness," Genes \& Development, vol. 20, no. 24, pp. 3426-3439, 2006. 
[11] J. Müller, O. Krijgsman, J. Tsoi et al., "Low MITF/AXL ratio predicts early resistance to multiple targeted drugs in melanoma," Nature Communications, vol. 5, no. 1, p. 5712, 2014.

[12] K. S. Hoek and C. R. Goding, "Cancer stem cells versus phenotype-switching in melanoma," Pigment Cell \& Melanoma Research, vol. 23, no. 6, pp. 746-759, 2010.

[13] C. Levy, M. Khaled, and D. E. Fisher, "MITF: master regulator of melanocyte development and melanoma oncogene," Trends in Molecular Medicine, vol. 12, no. 9, pp. 406-414, 2006.

[14] S. Carreira, J. Goodall, I. Aksan et al., "Mitf cooperates with $\mathrm{Rb} 1$ and activates $p 21^{\text {Cip1 }}$ expression to regulate cell cycle progression," Nature, vol. 433, no. 7027, pp. 764-769, 2005.

[15] Y. Cheli, S. Guiliano, T. Botton et al., "Mitf is the key molecular switch between mouse or human melanoma initiating cells and their differentiated progeny," Oncogene, vol. 30, no. 20, pp. 2307-2318, 2011.

[16] C. Köhler, D. Nittner, F. Rambow et al., "Mouse cutaneous melanoma induced by mutant BRaf arises from expansion and dedifferentiation of mature pigmented melanocytes," Cell Stem Cell, vol. 21, no. 5, pp. 679-693.e6, 2017.

[17] M. Zhang, J. S. di Martino, R. L. Bowman et al., “Adipocytederived lipids mediate melanoma progression via FATP proteins," Cancer Discovery, vol. 8, no. 8, pp. 1006-1025, 2018.

[18] C. W. Freudiger, W. Min, B. G. Saar et al., "Label-free biomedical imaging with high sensitivity by stimulated Raman scattering microscopy," Science, vol. 322, no. 5909, pp. 1857-1861, 2008.

[19] D. Zhang, M. N. Slipchenko, and J. X. Cheng, "Highly sensitive vibrational imaging by femtosecond pulse stimulated raman loss," Journal of Physical Chemistry Letters, vol. 2, no. 11, pp. 1248-1253, 2011.

[20] M. C. Fischer, J. W. Wilson, F. E. Robles, and W. S. Warren, "Invited review article: pump-probe microscopy," Review of Scientific Instruments, vol. 87, no. 3, p. 031101, 2016.

[21] D. Zhang, P. Wang, M. N. Slipchenko, and J. X. Cheng, "Fast vibrational imaging of single cells and tissues by stimulated Raman scattering microscopy," Accounts of Chemical Research, vol. 47, no. 8, pp. 2282-2290, 2014.

[22] S. Yue, M. N. Slipchenko, and J. X. Cheng, "Multimodal nonlinear optical microscopy," Laser and Photonics Reviews, vol. 5, no. 4, pp. 496-512, 2011.

[23] F. E. Robles, J. W. Wilson, M. C. Fischer, and W. S. Warren, "Phasor analysis for nonlinear pump-probe microscopy," Optics Express, vol. 20, no. 15, p. 17082, 2012.

[24] T.-Y. Chang, B.-L. Li, C. C. Y. Chang, and Y. Urano, "Acylcoenzyme A:cholesterol acyltransferases," American Journal of Physiology-Endocrinology and Metabolism, vol. 297, no. 1, pp. E1-E9, 2009.

[25] J. Li and J. X. Cheng, "Direct visualization of de novo lipogenesis in single living cells," Scientific Reports, vol. 4, p. 6807, 2015.

[26] K. Vriens, S. Christen, S. Parik et al., "Evidence for an alternative fatty acid desaturation pathway increasing cancer plasticity," Nature, vol. 566, no. 7744, pp. 403-406, 2019.

[27] F. Röhrig and A. Schulze, "The multifaceted roles of fatty acid synthesis in cancer," Nature Reviews Cancer, vol. 16, no. 11, pp. 732-749, 2016.

[28] C.-H. Yao, G.-Y. Liu, R. Wang, S. H. Moon, R. W. Gross, and G. J. Patti, "Identifying off-target effects of etomoxir reveals that carnitine palmitoyltransferase I is essential for cancer cell proliferation independent of $\beta$-oxidation," PLoS Biology, vol. 16, no. 3, pp. e2003782-e2003782, 2018.

[29] B. Raud, D. G. Roy, A. S. Divakaruni et al., "Etomoxir actions on regulatory and memory $\mathrm{T}$ cells are independent of Cpt1amediated fatty acid oxidation," Cell Metabolism, vol. 28, no. 3, pp. 504-515.e7, 2018.

[30] K. Reiss, I. Cornelsen, M. Husmann, G. Gimpl, and S. Bhakdi, "Unsaturated Fatty Acids Drive Disintegrin and Metalloproteinase (ADAM)-dependent Cell Adhesion, Proliferation, and Migration by Modulating Membrane Fluidity," Journal of Biological Chemistry, vol. 286, no. 30, pp. 26931-26942, 2011.

[31] N. Koundouros and G. Poulogiannis, "Reprogramming of fatty acid metabolism in cancer," British Journal of Cancer, vol. 122, no. 1, pp. 4-22, 2020.

[32] G. Pascual, A. Avgustinova, S. Mejetta et al., "Targeting metastasis-initiating cells through the fatty acid receptor CD36," Nature, vol. 541, no. 7635, pp. 41-45, 2017.

[33] K. M. Gharpure, S. Pradeep, M. Sans et al., "FABP4 as a key determinant of metastatic potential of ovarian cancer," Nature Communications, vol. 9, no. 1, p. 2923, 2018.

[34] K. M. Nieman, H. A. Kenny, C. V. Penicka et al., “Adipocytes promote ovarian cancer metastasis and provide energy for rapid tumor growth," Nature Medicine, vol. 17, no. 11, pp. 1498-1503, 2011.

[35] G. M. Alicea, V. W. Rebecca, A. R. Goldman et al., "Changes in aged fibroblast lipid metabolism induce age-dependent melanoma cell resistance to targeted therapy via the fatty acid transporter FATP2," Cancer Discovery, vol. 10, no. 9, pp. 1282-1295, 2020.

[36] H. M. Itkonen, M. Brown, A. Urbanucci et al., "Lipid degradation promotes prostate cancer cell survival," Oncotarget, vol. 8, no. 24, pp. 38264-38275, 2017.

[37] C.-k. Lee, S.-h. Jeong, C. Jang et al., "Tumor metastasis to lymph nodes requires YAP-dependent metabolic adaptation," Science, vol. 363, no. 6427, pp. 644-649, 2019.

[38] J. H. Park, S. Vithayathil, S. Kumar et al., "Fatty acid oxidationdriven Src links mitochondrial energy reprogramming and oncogenic properties in triple-negative breast cancer," Cell Reports, vol. 14, no. 9, pp. 2154-2165, 2016.

[39] B. Huang, B.-1. Song, and C. Xu, "Cholesterol metabolism in cancer: mechanisms and therapeutic opportunities," Nature Metabolism, vol. 2, no. 2, pp. 132-141, 2020.

[40] W. A. Freed-Pastor, H. Mizuno, X. Zhao et al., "Mutant p53 disrupts mammary tissue architecture via the mevalonate pathway," Cell, vol. 148, no. 1-2, pp. 244-258, 2012.

[41] G. Sorrentino, N. Ruggeri, V. Specchia et al., "Metabolic control of YAP and TAZ by the mevalonate pathway," Nature Cell Biology, vol. 16, no. 4, pp. 357-366, 2014.

[42] I. Y. Benador, M. Veliova, K. Mahdaviani et al., "Mitochondria bound to lipid droplets have unique bioenergetics, composition, and dynamics that support lipid droplet expansion," Cell Metabolism, vol. 27, no. 4, pp. 869-885.e6, 2018.

[43] R. J. Schulze, A. Sathyanarayan, and D. G. Mashek, "Breaking fat: the regulation and mechanisms of lipophagy," Biochimica et Biophysica Acta (BBA) - Molecular and Cell Biology of Lipids, vol. 1862, no. 10, pp. 1178-1187, 2017.

[44] A. Herms, M. Bosch, N. Ariotti et al., "Cell-to-cell heterogeneity in lipid droplets suggests a mechanism to reduce lipotoxicity," Current Biology, vol. 23, no. 15, pp. 1489-1496, 2013. 
[45] A. P. Bailey, G. Koster, C. Guillermier et al., "Antioxidant Role for Lipid Droplets in a Stem Cell Niche of Drosophila," Cell, vol. 163, no. 2, pp. 340-353, 2015.

[46] A. P. Velázquez, T. Tatsuta, R. Ghillebert, I. Drescher, and M. Graef, "Lipid droplet-mediated ER homeostasis regulates autophagy and cell survival during starvation," Journal of Cell Biology, vol. 212, no. 6, pp. 621-631, 2016.

[47] N. Dupont, S. Chauhan, J. Arko-Mensah et al., "Neutral lipid stores and lipase PNPLA5 contribute to autophagosome biogenesis," Current Biology, vol. 24, no. 6, pp. 609-620, 2014.

[48] J. X. Cheng and X. S. Xie, "Vibrational spectroscopic imaging of living systems: an emerging platform for biology and medicine," Science, vol. 350, no. 6264, article aaa8870, 2015.

[49] S. Yue, J. Li, S. Y. Lee et al., "Cholesteryl ester accumulation induced by PTEN loss and PI3K/AKT activation underlies human prostate cancer aggressiveness," Cell Metabolism, vol. 19, no. 3, pp. 393-406, 2014.

[50] J. Li, S. Condello, J. Thomes-Pepin et al., "Lipid desaturation is a metabolic marker and therapeutic target of ovarian cancer stem cells," Cell Stem Cell, vol. 20, no. 3, pp. 303-314.e5, 2017.

[51] K. C. Huang, J. Li, C. Zhang, Y. Tan, and J. X. Cheng, "Multiplex stimulated Raman scattering imaging cytometry reveals lipid-rich protrusions in cancer cells under stress condition," iScience, vol. 23, no. 3, article 100953, 2020.

[52] A. Ladanyi, A. Mukherjee, H. A. Kenny et al., "Adipocyteinduced CD36 expression drives ovarian cancer progression and metastasis," Oncogene, vol. 37, no. 17, pp. 2285-2301, 2018.

[53] A. Aloia, D. Müllhaupt, C. D. Chabbert et al., "A fatty acid oxidation-dependent metabolic shift regulates the adaptation ofBRAF-mutated melanoma to MAPK inhibitors," Clinical Cancer Research, vol. 25, no. 22, pp. 6852-6867, 2019.

[54] J. du, Y. Su, C. Qian et al., "Raman-guided subcellular pharmaco-metabolomics for metastatic melanoma cells," Nature Communications, vol. 11, no. 1, p. 4830, 2020.

[55] B. Peck and A. Schulze, "Lipid desaturation - the next step in targeting lipogenesis in cancer?," FEBS Journal, vol. 283, no. 15, pp. 2767-2778, 2016.

[56] L. Ge, J. S. Gordon, C. Hsuan, K. Stenn, and S. M. Prouty, "Identification of the $\Delta-6$ desaturase of human sebaceous glands: expression and enzyme activity," Journal of Investigative Dermatology, vol. 120, no. 5, pp. 707-714, 2003.

[57] Y. Vivas-García, P. Falletta, J. Liebing et al., "Lineage-restricted regulation of SCD and fatty acid saturation by MITF controls melanoma phenotypic plasticity," Molecular Cell, vol. 77, no. 1, pp. 120-137.e9, 2020.

[58] M. Triki, G. Rinaldi, M. Planque et al., "mTOR signaling and SREBP activity increase FADS2 expression and can activate sapienate biosynthesis," Cell Reports, vol. 31, no. 12, article 107806, 2020.

[59] K. Willert, J. D. Brown, E. Danenberg et al., "Wnt proteins are lipid-modified and can act as stem cell growth factors," Nature, vol. 423, no. 6938, pp. 448-452, 2003.

[60] J. Jeong and A. P. McMahon, "Cholesterol modification of hedgehog family proteins," Journal of Clinical Investigation, vol. 110, no. 5, pp. 591-596, 2002.

[61] A. T. Weeraratna, Y. Jiang, G. Hostetter et al., "Wnt5a signaling directly affects cell motility and invasion of metastatic melanoma," Cancer Cell, vol. 1, no. 3, pp. 279-288, 2002.

[62] C. S. Liao, P. Wang, C. Y. Huang et al., "In Vivoandin SituSpectroscopic imaging by a handheld stimulated Raman scattering microscope," ACS Photonics, vol. 5, no. 3, pp. 947-954, 2018.

[63] G. Zhou, O. Soufan, J. Ewald, R. E. W. Hancock, N. Basu, and J. Xia, "NetworkAnalyst 3.0: a visual analytics platform for comprehensive gene expression profiling and meta-analysis," Nucleic Acids Research, vol. 47, no. W1, pp. W234-W241, 2019.

[64] Y. Zhou, B. Zhou, L. Pache et al., "Metascape provides a biologist-oriented resource for the analysis of systems-level datasets," Nature Communications, vol. 10, no. 1, p. 1523, 2019.

[65] E. G. Bligh and W. J. Dyer, "A rapid method of total lipid extraction and purification," Canadian Journal of Biochemistry and Physiology, vol. 37, no. 8, pp. 911-917, 1959. 